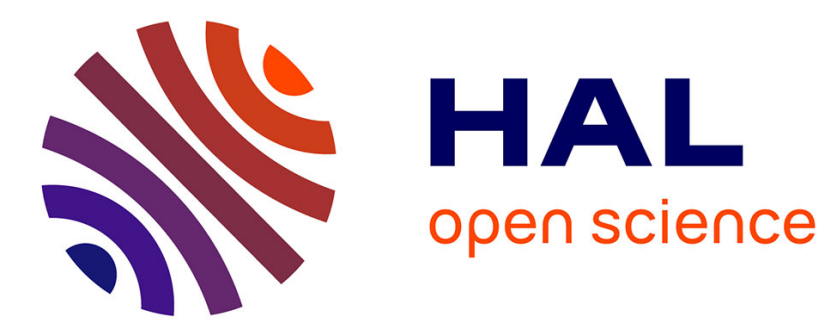

\title{
Structural energy flow optimization through adaptive shunted piezoelectric metacomposites
}

\author{
Manuel Collet, Morvan Ouisse, Mohamed Ichchou
}

\section{To cite this version:}

Manuel Collet, Morvan Ouisse, Mohamed Ichchou. Structural energy flow optimization through adaptive shunted piezoelectric metacomposites. Journal of Intelligent Material Systems and Structures, 2012, 23 (15), pp.1661-1677. 10.1177/1045389X12449915 . hal-02300118

\section{HAL Id: hal-02300118 \\ https://hal.science/hal-02300118}

Submitted on 3 Feb 2020

HAL is a multi-disciplinary open access archive for the deposit and dissemination of scientific research documents, whether they are published or not. The documents may come from teaching and research institutions in France or abroad, or from public or private research centers.
L'archive ouverte pluridisciplinaire HAL, est destinée au dépôt et à la diffusion de documents scientifiques de niveau recherche, publiés ou non, émanant des établissements d'enseignement et de recherche français ou étrangers, des laboratoires publics ou privés. 


\title{
Structural energy flow optimization through adaptive shunted piezoelectric metacomposites
}

\author{
M Collet, M. Ouisse \\ FEMTO-ST, Applied Mechanics Department, \\ UMR-CNRS 6174, \\ 24 chemin de l'épitaphe, \\ 25000 Besançon, France \\ M. N. Ichchou \\ LTDS UMR5513 \\ Ecole Centrale de Lyon \\ 36 avenue Guy de Collongue \\ 69134 Ecully, France
}




\begin{abstract}
In this paper, a numerical approach for modeling and optimizing 2D smart metacomposites is presented. The proposed methodology is based on the Floquet-Bloch theorem in the context of elastodynamics including distributed shunted piezoelectric patches. The dedicated numerical technique is able to cope with the multi-modal wave dispersions behaviour over the whole first Brillouin zone for periodically distributed 2D shunted piezo-mechanical systems. Some indicators allowing the optimization of the shunt impedance for specific performance objectives are presented, and applied for illustration purposes on the design of an adaptive metacomposite with specific functionalities. In order to validate the strategy, the designed metacomposite is integrated in a support structure, and a full 3D model is derived to illustrate the efficiency of the approach. This work is a collaborative effort supported by the French Research Agency under grant number NT09-617542.
\end{abstract}

Keywords : Adaptive Metamaterial, Distributed Shunted piezoelectric patches, Wave propagation optimization 


\section{Introduction}

The design of innovative structures with high degree of performances is a permanent challenge for both industrial and academic communities. The issue is often posed in many engineering sectors such as civil engineering and transportations (ground and aeronautics). In the transportation sector the question becomes drastic with regards to energy savings concerns and with respect to sustainable developments (see reports of Clean Sky and DREAM EU Projects, AIAA's Emerging Technologies Committee - ETC). This is the case, for instance, in the aeronautic sector where a fantastic effort is made in order to integrate lightweight structures in the design of new aircrafts. Advanced materials and structures such as composite materials are thus more and more used. Such composite materials make the structures lighter and stiffer. They are now intensively included in the design and manufacturing of new aircrafts and space shuttles. However, such composite structures bring new vibration and acoustic problems. Indeed, compared to metallic structures it is noticeable that composites structures evolves specific noise emissions and particular safety issues. The majority of the projects dedicated to smart materials have focused on adding smart functionalities to existing structures - piezoelectric patches to plates and shells for acoustic or structural health monitoring as an example. Nonetheless, these types of solutions tend to address efficiently only one particular problem at the time, and require increased complexity in terms of wiring, controllers and modifications of the baseline structure to tackle successfully structural, acoustic control and structural health monitoring. Hence, among the problems to be considered, the broadband nature (from the low frequency LF up to the high frequency HF and including the mid frequency MF range) of vibration and noise associated to composite structures and their assembly is still an open and challenging concern. This paper is a contribution to the challenges of designing, optimizing and manufacturing a new class of truly integrated and intrinsic smart active metamaterial, structures and systems, also called metacomposite with ground breaking engineering performance in terms of mechanical and vibroacoustics behaviour.

The concept of metacomposite has a specific meaning in this paper. This concept couples two different aspects in vibration control. The first concept is connected to periodic structures theories usually connected to metamaterial developments. In this case, it is well known that the dynamic behaviour is fully connected to periodicity ratios and existing pass bands and blocked bands can be of real use in vibration control. The second concept is associated to vibration control through piezoelectric and smart materials. Specifically, shunted piezoelectric smart materials are employed for the metacomposite achievement by integrating into the metamaterial electronics and numerical components allowing implementation of adaptive and controlled behaviour. We also tend to extend the notion of programmable matter within the meaning of work presented in to vibroacoustic programming. The paper main novelty is then the design through full numerical analysis of a smart structure with broad band control abilities. Wave based methods and numerical simulation tools are adapted to the proposed concept. The realized composite controlled dynamic is in the LF low frequency and mid frequency MF band as well. Development of a wave trap metacomposite can be the ultimate goal of the reported research and results.

Looking at the literature, there are intensive involvements in both the modelling and control communities. Indeed, researchers studied numerous methods to deal with undesirable vibrations and noise. These approaches can be generally classified as three catalogues which are passive control, active control and semi-active or semi-passive control. Several passive control ways were developed. Tuned mass damper (TMD) or harmonic absorber is one of the most popular passive control approach used since many years in many different engineering applications (Ormondroyd \& Den Hartog 1928, Den Hartog 1956, Spencer \& Nagarajaiah 2003). The corresponding device is normally mounted onto a host structure to absorb the structural mechanical energy when the structure vibrates at its 
resonant frequency. Passive damping technologies can also be considered as passive control strategies especially when optimization design is carried out (Milman \& Cheng-Chih 1994, Baz 1997). The main disadvantages of these passive strategies are also their narrow band of efficiency and the necessity to introduce massive elements (damping materials or massive absorber) inside the considered structure. Optimal efficiency in low and broad frequency bands can be reached by using active technologies for vibration or noise control (Preumont 2002, Nelson \& Elliott 1992, Banks, Smith \& Wang 1996) with the drawback of introducing complex and massive numerical and electronics elements and external power supply systems.

Semi-active control or semi-passive control possesses partial advantages of both passive and active control. Piezoelectric shunt technologies are promising passive techniques for vibration control of flexible structures. Contrary to active control, the only external element to be used is a Passive Electrical Network (PEN) that is directly connected to the electrodes of the piezoelectric device. With this approach, the sensing element is not needed and the use of a passive network guarantees the stability of the coupled system. Furthermore, shunted piezoelectric systems offer a very attractive path for electro-mechanical integration and distribution. Numerous works have been published (Park \& Palumbo 2004, Becker, Fein, Maess \& Gaul 2006, Hurlebaus \& Gaul 2006, Hagood \& von Flotow 1991, Agnes 1994, dell'Isola, Maurini \& Porfiri 2004, Moheimani \& Fleming 2006, Cross \& Fleeter 2002, Beck, Cunefare \& Ruzzene 2008a) that present analyses of the capability and efficiency of a single shunted piezoelectrical patch for structural stabilization and wave cancellation. Hagood and VonFlotow (Hagood \& von Flotow 1991) provided the first analytical formulation for passive shunt networks. They demonstrated how a piezoelectric patch shunted through a single resistive-inductive $(\mathrm{RL})$ circuit acts as a vibration absorber tuned at the resonance frequency of the circuit. Since then, more complex shunting circuits have been investigated to extend the effectiveness over broader frequency bands: multi-mode techniques (Wu 1998, Wu \& Bicos 1997), state switching (Corr \& Clark 2002), synthetic impedance (Fleming, Belirens \& Moheimani 2000) or negative impedance circuits (Behrens, Fleming \& Moheimani 2001, Park \& Palumbo 2004, Park \& Baz 2005, Fukada, Date \& Kimura 2002, Neubauer, Oleskiewicz, Popp \& Krzyzynski 2006). Park and Palumbo decomposed the wave field on a beam to find a minimization of the reflected wave component (Park \& Palumbo 2004). By noticing the link between the wave based approach of Park and Palumbo and the power input to the system, Cunefare developed a parameter selection technique based on the reactive input power (Cunefare 2006). Periodic structures have been shown to improve the broadband performance of control systems on structures (Casadei, Beck, Ruzzene \& Cunefare 2009, Thorp, Ruzzene \& Baz 2001, Thorp, Ruzzene \& Baz 2005). On all these works, piezoelectric shunt damping also appears as promising passive or semi-passive technique for vibration control of flexible structures (Beck et al. 2008a, Cunefare 2006). Contrary to active control, the only external element to be used is a passive electrical network (PEN) that is directly connected to the electrodes of the piezoelectric device and can be integrated into the composite material (Forward 1978, Fleming, Behrens \& Moheimani 2003, Fleming \& Moheimani 2004). In this paper, thus shunted piezoelectric concept will be developed further for wave control purposes mainly. Unlike most of the existing papers, this contribution will concentrate on a deep multi-physical numerical modelling of composite structures coupled to shunted piezoelectric patches based on mathematical approach developed in (Collet, Ouisse, Ruzzene $\&$ Ichchou 2011). The numerical investigation is concerned with $2 \mathrm{D}$ wave treatment and optimization in such metacomposite. This is also an original aspect of the paper.

Considering numerical simulation of wave behaviours in elastodynamic media, two main strategies can be reported: the semi-analytical finite element (SAFE) and the wave finite element method (WFE). Both are of spectral nature but of different philosophies and practical use. Semi-analytical finite element method was investigated by many researchers in the past decades. This method was first proposed by (Nelson \& Dong 1973) for plate waves to analyse natural vibrations of lam- 
inated orthotropic plates in which lamina is discretized by finite element. The (cross-sectionally) two-dimensional problem was first developed by (Aalami 1973), which can be extended to the wave guides of arbitrary cross-sectional shape. Recently, Gavric calculated the dispersion relationship in a free rail by using triangular and quadrilateral elements, with those elements obtained from HamiltonŠs principle (Gavric 1995). Hayashi et al. (Hayashi, Song \& Rose 2003) derived the SAFE formulation through virtual work principles and proposed a way to calculate the group velocity using the eigensolution at a given frequency. Damljanovic and Weaver (Damljanovic \& Weaver 2004) developed the linear triangular elements for SAFE method using Lagrange equations to investigate the elastic waves in wave guides of arbitrary cross-section. Bartoli et al. (Bartoli, Marzani \& Viola 2006) examined the wave mode characteristics in damped waveguides by using SAFE method, where the elements were also derived from Hamilton's principle. This method has also been adopted to investigate the wave propagation characteristics for thin-walled structures (Finnveden 2004), where the polynomial interpolation is used in the propagation axis and thus leads to the polynomial eigenvalue problems. The method is extended to curved structures in (Finnveden \& Fraggstedt 2008), where an isoparametric element is used.

Another spectral method has attracted many researchers, which is based on the technique dealing with the wave propagation problems of periodic structures. Mead (Mead 1973) proposed a general theory in order to determine harmonic wave propagation characters, where both one-dimensional and two-dimensional periodic systems are considered. Recently, more studies focus on extending this idea to homogeneous structures rather than the generally periodic systems comprised of an arbitrary substructure (see (Houillon, Ichchou \& Jezequel 2005, Bocquillet, Ichchou \& Jezequel 2003)). This Wave Finite Element (WFE) method regards the homogeneous wave guide structure as a periodic system assembled by identical substructures. Thus the method for periodic systems can be seamlessly transplanted to the WFE method for eigenmode extraction. The identical substructures can be discretized with the aid of some commercial FE procedures, rather than the development of a relatively new FE code for specific elements. This allows the existing element libraries and powerful grid generation procedures to be used for many engineering structures. The WFE method is easy to apply due to its perfect connection with the standard FE method. This method has been used for structural vibration analyses (Mace, Duhamel, Brennan \& Hinke 2005, Zhou, Ichchou \& Mencik 2009, Hardy, Ichchou, Jezequel \& Trentin 2009, Duhamel, Mace \& Brennan 2006, Houillon et al. 2005), wave propagation in elastic wave guides (Mencik \& Ichchou 2005b, Chen \& Wilcox 2007), and calculations of the Green's functions for homogeneous acoustical and elastic media (Duhamel 2007). The method was also implemented for wave propagation and dynamic problems in homogeneous structures with internal fluid (Bocquillet et al. 2003, Mencik \& Ichchou 2007, Maess, Wagner \& Gaul 2006), where the studies were based on the WFE method for the one-dimensional wave propagation problem and concentrated on the lower frequency problems (far below the frequency of interest for NDT). One of the main problems all these approaches are facing is the difficulty to compute the damped wave numbers in complex media. Precisely, wave properties in the whole Brillouin domain for multiphysics problem incorporating shunted piezoelectric patches is not an easy task. This involvement is necessary for vibroacoustic behaviour optimization purposes of such periodic smart structures (Collet, Ouisse, Ruzzene \& Ichchou 2011, Collet, Ouisse, Ichchou \& Ruzzene 2011).

The objectives of the work reported in this document were threefold. First, the paper offers a suited numerical approach for computing wave dispersion in smart multiphysics metacomposite with shunted piezoelectric patches. Second, the achieved model is extensively used for electromechanical optimization studies of the metacomposite, and finally, the optimized configuration is used in a finite structure to check the applicability of the concept on a practical application. The paper is structured in four different sections. After recalling the Floquet-Bloch theorems in section 2, we introduce a numerical formulation to compute the multi-modal damped wave numbers dispersion 
in the whole first Brillouin domain. Based on this wave modelling, optimization of the electrical impedance of the shunted circuit is made in order to control group velocity of flexural waves or to increase damping induced by the electric circuit. The corresponding optimal impedances are then tested for controlling the mid frequency (MF) response of a semi-distributed finite system. The numerical achievements prove the effectiveness of the proposed metacomposite concept and draw future steps and perspectives.

\section{Piezo-Elasto Dynamical Application of the Floquet-Bloch Theo-}

\section{rem}

In this section the application of the celebrated Floquet-Bloch theorem is presented for piezoelastodynamic problems. Based on the well known results obtained by Floquet (Floquet 1883) for monodimensional problems and later rediscovered by Bloch (Bloch 1928) in multidimensional problems, an original application to bi-dimensional piezo-elastodynamical problem has been proposed recently (Collet, Ouisse, Ichchou \& Ruzzene 2010). This formulation leads to very general numerical implementation for computing waves dispersion for periodically smart distributed mechanical systems incorporating electronic components, damping effects or any frequency-dependent characteristics. The main ideas of the approach are recalled here, together with the specific points related to the inclusion of piezoelectric effects in the model.

\subsection{The Bloch theorem}

The Bloch theorem, in its original version, gives the form of homogeneous states of Schrödinger equation with periodic potential. This theorem can be considered as a multidimensional application of the Floquet theorem (Joannopoulos, Meade \& Winn 1995). The periodic medium (or potential) properties satisfy $M(\boldsymbol{x}+R \cdot \boldsymbol{m})=M(\boldsymbol{x}), \boldsymbol{m} \in \mathbb{Z}^{3}$ where $R=\left[\boldsymbol{r}_{1}, \boldsymbol{r}_{2}, \boldsymbol{r}_{3}\right] \in \mathbb{R}^{3 \times 3}$ is a matrix grouping the three lattice's basis vectors (in $3 \mathrm{D}$ ). The primitive cell is defined as a convex polyhedron of $\mathbb{R}^{3}$ called $\Omega_{x}$. The reciprocal unit cell is denoted by $\Omega_{k}$ and limited by the reciprocal lattice vector defined by the three vectors $\boldsymbol{g}_{j}$ so that: $\boldsymbol{r}_{i} . \boldsymbol{g}_{j}=2 \pi \delta_{i j}\left(\delta_{i j}\right.$ being the Kronecker index). If $\Omega_{x}$ is the irreductible primitive cell, $\Omega_{k}$ corresponds to the first Brillouin zone of the lattice (Kittel 1986).

The Bloch Theorem stipulates that any function $\boldsymbol{u}(\boldsymbol{x}) \in L^{2}\left(\mathbb{R}^{3}, \mathbb{C}^{n}\right)$ can be expressed as

$$
\boldsymbol{u}(\boldsymbol{x})=\int_{\Omega_{k}} e^{i \boldsymbol{k} \boldsymbol{x}} \tilde{\boldsymbol{u}}(\boldsymbol{x}, \boldsymbol{k}) d \boldsymbol{k}
$$

where the Bloch amplitude $\tilde{\boldsymbol{u}}(\boldsymbol{x}, \boldsymbol{k})$ is $\Omega_{x}$-periodic and has the representations

$$
\begin{aligned}
\tilde{\boldsymbol{u}}(\boldsymbol{x}, \boldsymbol{k}) & =\sum_{\boldsymbol{n} \in \mathbb{Z}^{3}} \hat{\boldsymbol{u}}(\boldsymbol{k}+G \boldsymbol{n}) e^{i G \boldsymbol{n} \cdot \boldsymbol{x}} \\
& =\frac{\left|\Omega_{x}\right|}{(2 \pi)^{3}} \sum_{\boldsymbol{n} \in \mathbb{Z}^{3}} \boldsymbol{u}(\boldsymbol{x}+R \boldsymbol{n}) e^{i \boldsymbol{k}(\boldsymbol{x}+R \boldsymbol{n})}
\end{aligned}
$$

where $\hat{\boldsymbol{u}}(\boldsymbol{k})$ stands for the Fourier transform of $\boldsymbol{u}(\boldsymbol{x})$ and $G=\left[\boldsymbol{g}_{1}, \boldsymbol{g}_{2}, \boldsymbol{g}_{3}\right]$ is the reciprocal lattice matrix in the later. It can also be demonstrated that the mean value of the Bloch amplitude is the Fourier amplitude of $\boldsymbol{u}(\boldsymbol{x})$ for the corresponding wave vector: $\langle\tilde{\boldsymbol{u}}(., \boldsymbol{k})\rangle_{\Omega_{x}}=\hat{\boldsymbol{u}}(\boldsymbol{k})$. Using the 
Bloch theorem to represent the solutions of periodical partial derivative equations implies that all derivatives are shifted by $\boldsymbol{k}$ in the sense given by the spatial operator.

Based on that theorem, the expansion functions $\boldsymbol{v}_{m}(\boldsymbol{x}, \boldsymbol{k})$ can be defined. They are called the Bloch eigen modes, and can be used to represent the Bloch amplitudes of any solution of the corresponding partial derivative equation as

$$
\tilde{\boldsymbol{u}}(\boldsymbol{x}, \boldsymbol{k})=\sum_{m} \boldsymbol{u}_{m}(\boldsymbol{k}) \boldsymbol{v}_{m}(\boldsymbol{x}, \boldsymbol{k})
$$

and at the same time diagonalize the partial derivative equations. The expansion coefficients $\boldsymbol{u}_{m}(\boldsymbol{k})$ depend on the disturbance and on the induced wave vector (see (Bensoussan, Lions \& Pananicolaou 1978) for details).

\subsection{Application to piezo-elastodynamic}

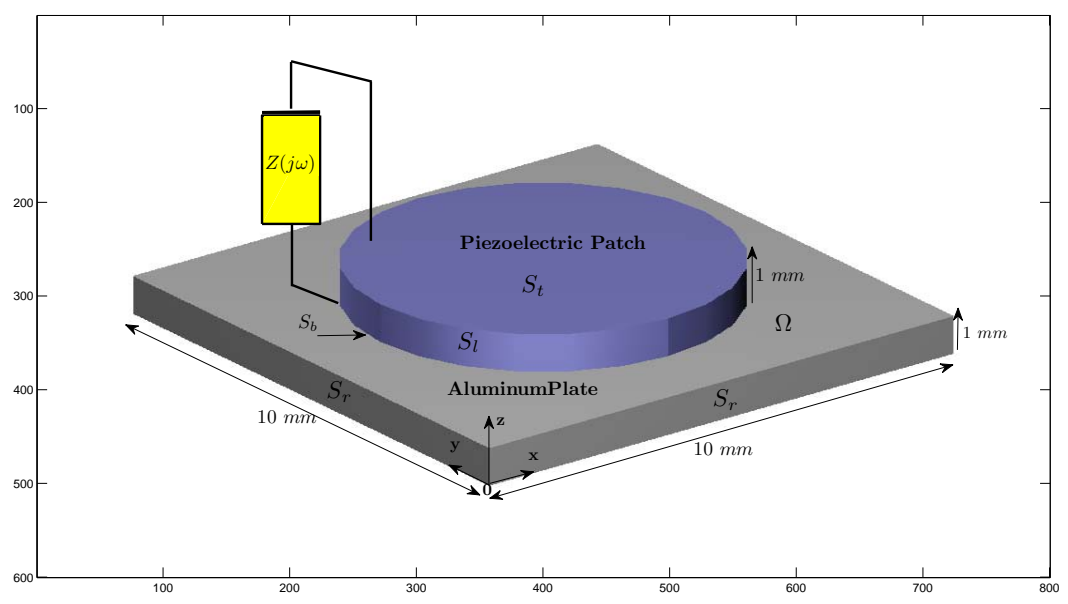

Figure 1: Generic 3D piezocomposite periodic cell

Let us consider a piezo-elastodynamic problem made of infinite periodic distribution of unitary cell described in figure 1. The harmonic homogeneous dynamical equilibrium of system is driven by the following partial derivative equations $(t \in \mathbb{R})$ :

$$
\begin{cases}\rho \ddot{\boldsymbol{w}}(\boldsymbol{x}, t)-\nabla \sigma(\boldsymbol{x}, t)=0 & \forall \boldsymbol{x} \in \Omega_{x} \\ -\nabla D(\boldsymbol{x}, t)=0 & \forall \boldsymbol{x} \in \Omega_{x}\end{cases}
$$

where $\boldsymbol{w}(\boldsymbol{x}, t) \in \mathbb{R}^{3}\left(\Omega_{x}, \mathbb{R}\right)$ is the displacement vector, $\sigma$ represents the Cauchy stress tensor, $\epsilon=$ $\nabla_{\text {sym }} \boldsymbol{w}=\frac{1}{2}\left(\nabla \boldsymbol{w}^{T}(\boldsymbol{x}, t)+\boldsymbol{w}(\boldsymbol{x}, t) \nabla^{T}\right)$ the Green strain tensor, $D(\boldsymbol{x}, t)$ the electric displacement. The linear constitutive material behavior relationships can be written as

$$
\begin{aligned}
\sigma & =C_{E}(\boldsymbol{x}) \epsilon-e^{T}(\boldsymbol{x}) \boldsymbol{E} \\
\boldsymbol{D} & =e(\boldsymbol{x}) \epsilon+\varepsilon_{S}(\boldsymbol{x}) \boldsymbol{E}
\end{aligned}
$$


where $\boldsymbol{E}=-\nabla V$ is the electric field vector ( $V$ being the voltage), $C_{E}$ the elasticity tensor at constant electrical field, $e^{T}$ the piezoelectric coupling tensor and $\varepsilon_{S}$ the dielectric permittivity at constant strain. We add to this set of equilibrium equations an output expression

$$
q^{o}=-\int_{S_{t}} \boldsymbol{D} \cdot \boldsymbol{n} d S
$$

allowing the introduction of the charge measurement on the piezoelectric's top electrode and hence the dual counterpart of the imposed electrical Dirichlet boundary condition for applying the shunt impedance operator.

The equations above are consistent for each kind of material to the extent that null piezoelectric and permittivity tensors can be used when passive materials are considered. All of these tensors also depend on the spatial location vector $\boldsymbol{x}$ and are $\Omega_{x}$ periodic. By applying a Fourier transformation, the piezo-elastodynamic equilibrium can also be written as $(\omega \in \mathbb{R})$ :

$$
\begin{aligned}
\rho \omega^{2} \boldsymbol{w}(\boldsymbol{x}, \omega)+\nabla C \nabla_{\text {sym }}(\boldsymbol{w}(\boldsymbol{x}, \omega))+\nabla e^{T}(\boldsymbol{x}) \nabla V(\boldsymbol{x}, \omega) & =0 \forall \boldsymbol{x} \in \Omega_{x} \\
-\nabla e(\boldsymbol{x}) \nabla_{\text {sym }}(\boldsymbol{w}(\boldsymbol{x}, \omega))+\nabla \varepsilon_{S}(\boldsymbol{x}, \omega) \nabla V(\boldsymbol{x}, \omega) & =0 \forall \boldsymbol{x} \in \Omega_{x}
\end{aligned}
$$

As the problem is $2 \mathrm{D}$ infinitely periodic, mechanical boundary conditions are included in the formulation, while electrostatic boundary conditions have to be considered on each cell $(\omega \in \mathbb{R})$ :

$$
\begin{cases}V(\boldsymbol{x}, \omega)=0 & \forall \boldsymbol{x} \in S_{b} \\ V(\boldsymbol{x}, \omega)=V^{o}(\omega) & \forall \boldsymbol{x} \in S_{t} \\ \boldsymbol{D} \boldsymbol{n}(\boldsymbol{x}, \omega)=0 & \forall \boldsymbol{x} \in S_{l}\end{cases}
$$

where $S_{b}$ is the grounded bottom electrode of the piezoelectric layer, $S_{t}$ is the top electrode connected to the external shunt and $s_{l}$ the lateral electrode. The top electrode applied feedback voltage $V^{o}$ depends on the shunt characteristic and on the collected charges $q^{o}$ given in eq. (7) and can be expressed in the Fourier space by:

$$
V^{o}(\omega)=-Z(\omega) q^{o}(\omega)
$$

In the following, the frequency dependency on $\omega$ of each functions in the Fourier space is removed for simplifying the notations. Considering a primitive cell of the periodic problem $\Omega_{x}$, the Bloch eigenmodes (3) and the dispersion functions can be computed by searching the eigen solutions of the homogeneous problem (8) and (9) with mechanical periodic boundary conditions and electric ones given by (10) as:

$$
\boldsymbol{u}(\boldsymbol{x})=\left[\begin{array}{c}
\boldsymbol{w}(\boldsymbol{x}) \\
V(\boldsymbol{x})
\end{array}\right]=\boldsymbol{u}_{n, \boldsymbol{k}}(\boldsymbol{x}) e^{i \boldsymbol{k} \cdot \boldsymbol{x}}
$$

where $\boldsymbol{u}_{n, \boldsymbol{k}}(\boldsymbol{x})=\left[\begin{array}{c}\boldsymbol{w}_{n, \boldsymbol{k}}(\boldsymbol{x}) \\ V_{n, \boldsymbol{k}}(\boldsymbol{x})\end{array}\right]$ is composed of $\Omega_{x}$ periodic functions. By introducing expression (12) in the piezo-elastodynamic equations (8) and (9), $\boldsymbol{w}_{n, \boldsymbol{k}}(\boldsymbol{x}), V_{n, \boldsymbol{k}}(\boldsymbol{x})$ and $\omega_{n}(\boldsymbol{k})$ can be found by solving the generalized eigenvalues problem:

$$
\begin{aligned}
0= & \rho \omega_{n}^{2}(\boldsymbol{k}) \boldsymbol{w}_{n, \boldsymbol{k}}(\boldsymbol{x})+\nabla C \nabla_{s y m} \boldsymbol{w}_{n, \boldsymbol{k}}(\boldsymbol{x})+\nabla e^{T} \nabla V_{n, \boldsymbol{k}}(\boldsymbol{x}) \\
& +i k\left[\left(C \nabla_{s y m} \boldsymbol{w}_{n, \boldsymbol{k}}(\boldsymbol{x})\right) . \boldsymbol{\Phi}+\nabla\left(C \Xi_{n, \boldsymbol{k}}(\boldsymbol{x})\right)\right] \\
& +i k\left[\left(\nabla e^{T} V_{n, \boldsymbol{k}}(\boldsymbol{x})\right) . \boldsymbol{\Phi}\left(e^{T} \nabla V_{n, \boldsymbol{k}}(\boldsymbol{x})\right) . \boldsymbol{\Phi}\right] \\
& -k^{2}\left[\left(C \Xi_{n, k}(\boldsymbol{x})\right) . \boldsymbol{\Phi}+V_{n, \boldsymbol{k}}(\boldsymbol{x})\left(e^{T} \boldsymbol{\Phi}\right) . \boldsymbol{\Phi}\right] \forall \boldsymbol{x} \in \Omega_{x}, \\
0 & =-\nabla e \nabla_{s y m} \boldsymbol{w}_{n, \boldsymbol{k}}(\boldsymbol{x})+\nabla \varepsilon_{S} \nabla V_{n, \boldsymbol{k}}(\boldsymbol{x}) \\
& -i k\left[\nabla\left(e \Xi_{n, \boldsymbol{k}}(\boldsymbol{x})\right)+\left(e \nabla_{s y m}\left(\boldsymbol{w}_{n, \boldsymbol{k}}(\boldsymbol{x})\right)\right) . \boldsymbol{\Phi}\right] \\
& +i k\left[\left(\nabla \varepsilon_{S} V_{n, \boldsymbol{k}}(\boldsymbol{x})\right) . \boldsymbol{\Phi}+\left(\varepsilon_{S} \nabla V_{n, \boldsymbol{k}}\right)(\boldsymbol{x}) . \boldsymbol{\Phi}\right] \\
& +k^{2}\left[\left(e \Xi_{n, \boldsymbol{k}}(\boldsymbol{x})\right) . \boldsymbol{\Phi}-\left({ }_{S} \boldsymbol{\Phi} V_{n, \boldsymbol{k}}(\boldsymbol{x})\right) . \boldsymbol{\Phi}\right] \forall \boldsymbol{x} \in \Omega_{x},
\end{aligned}
$$


with the associated boundary conditions:

$$
\begin{cases}\boldsymbol{w}_{n, \boldsymbol{k}}(\boldsymbol{x}-R . \boldsymbol{m})=\boldsymbol{w}_{n, \boldsymbol{k}}(\boldsymbol{x}) & \forall \boldsymbol{x} \in S_{r} \boldsymbol{m} \in \mathbb{Z}^{2}, \\ V_{n, \boldsymbol{k}}(\boldsymbol{x})=0 & \forall \boldsymbol{x} \in S_{b}, \\ V_{n, \boldsymbol{k}}(\boldsymbol{x})=-Z(i \omega) q_{n, \boldsymbol{k}}^{o} & \forall \boldsymbol{x} \in S_{t} \\ \boldsymbol{D} \boldsymbol{n}=0 & \forall \boldsymbol{x} \in S_{l} .\end{cases}
$$

In these equations $\boldsymbol{k}=k\left[\begin{array}{c}\cos (\phi) \\ \sin (\phi) \\ 0\end{array}\right]=k \boldsymbol{\Phi}$ where $\phi$ represents the direction angles into the reciprocal 2D lattice domain and $\Xi_{n, \boldsymbol{k}}(\boldsymbol{x})=\frac{1}{2}\left(\boldsymbol{w}_{n, \boldsymbol{k}}(\boldsymbol{x}) \cdot \boldsymbol{\Phi}^{T}+\boldsymbol{\Phi} \cdot \boldsymbol{w}_{n, \boldsymbol{k}}^{T}(\boldsymbol{x})\right)$ is the symmetric dyadic tensor or the dyadic product of the displacement $\boldsymbol{w}_{n, \boldsymbol{k}}(\boldsymbol{x})$ and direction vector $\boldsymbol{\Phi} . S_{r}$ are the interfaces of the cells continuum, and $R$ is the matrix grouping the two lattice's basis vectors (in $2 \mathrm{D}$ in the considered problem). In the electrical boundary conditions, $q_{n, \boldsymbol{k}}^{o}$ is given by:

$$
\begin{aligned}
q_{n, \boldsymbol{k}}^{o}=\int_{S_{t}}[- & e \nabla_{s y m} \boldsymbol{w}_{n, \boldsymbol{k}}(\boldsymbol{x})+i k \nabla e \Xi_{n, \boldsymbol{k}}(\boldsymbol{x}) \\
& \left.+\varepsilon_{S}\left(\nabla V_{n, \boldsymbol{k}}(\boldsymbol{x})+i k V_{n, \boldsymbol{k}}(\boldsymbol{x}) \boldsymbol{\Phi}\right)\right] . \boldsymbol{n} d S
\end{aligned}
$$

where $\boldsymbol{n}$ is the surface outpointing unitary normal vector.

The proposed formulation is then based on the computation of the Floquet vectors given in equations (13) and (14)), instead of computing the Floquet propagators commonly used for elastodynamic applications. The full 2D waves dispersions functions can then be obtained, while damping and electrical impedance can clearly be introduced into the piezo-elastodynamic operator. The adopted methodology allows the computation of the complete complex map of the dispersion curves incorporating computation of evanescent waves and allowing the introduction of damping and shunt operator if any.

\subsection{Weak formulation and computation of waves dispersion functions in peri-}

\section{odical piezo-composite lattice}

Let us consider the partial derivative equations (13), (14) on a unit cell $\Omega$. It stands for a generalized eigenvalue problem leading to compute the dispersion functions $\omega_{n}(\boldsymbol{k})$ and the corresponding Floquet eigenvectors $\boldsymbol{u}_{n, \boldsymbol{k}}(\boldsymbol{x})$. For computing the 2D dispersions curves, we need to introduce a suitable weak formulation.

If $\boldsymbol{u}_{n, \boldsymbol{k}}(\boldsymbol{x})$ is a solution of equations (13), (14), then:

$$
\begin{aligned}
& \forall \tilde{\boldsymbol{w}}_{n, \boldsymbol{k}}(\boldsymbol{x}) \in\left\{H_{1}\left(\Omega, \mathbb{C}^{3}\right) / \tilde{\boldsymbol{w}}_{n, \boldsymbol{k}}(\boldsymbol{x}-R \boldsymbol{m})=\tilde{\boldsymbol{w}}_{n, \boldsymbol{k}}(\boldsymbol{x}) \forall \boldsymbol{x} \in S_{r}\right\} \text { and } \\
& \forall \tilde{V}_{n, \boldsymbol{k}}(\boldsymbol{x}) \in\left\{H_{1}(\Omega, \mathbb{C}) / \tilde{V}_{n, k}(\boldsymbol{x})=0 \forall \boldsymbol{x} \in S_{b} \text { and } \tilde{V}_{n, k}(\boldsymbol{x})=V^{o} \forall \boldsymbol{x} \in S_{t}\right\} \text { one has: } \\
& 0=\int_{\Omega}\left[\rho \omega_{n}^{2}(\boldsymbol{k}) \tilde{\boldsymbol{w}}_{n, \boldsymbol{k}}(\boldsymbol{x}) \boldsymbol{w}_{n, \boldsymbol{k}}(\boldsymbol{x})\right. \\
& -\left(\tilde{\varepsilon}_{n, \boldsymbol{k}}(\boldsymbol{x})-i k \tilde{\Xi}_{n, \boldsymbol{k}}(\boldsymbol{x})\right) C\left(\varepsilon_{n, \boldsymbol{k}}(\boldsymbol{x})+i k \Xi_{n, \boldsymbol{k}}(\boldsymbol{x})\right) \\
& +\left(\tilde{\varepsilon}_{n, \boldsymbol{k}}(\boldsymbol{x})-i k \tilde{\Xi}_{n, \boldsymbol{k}}(\boldsymbol{x})\right) e^{T}\left(\nabla V_{n, \boldsymbol{k}}(\boldsymbol{x})+i k V_{n, \boldsymbol{k}}(\boldsymbol{x}) \boldsymbol{\Phi}\right) \\
& -\left(\nabla \tilde{V}_{n, \boldsymbol{k}}(\boldsymbol{x})-i k \tilde{V}_{n, \boldsymbol{k}}(\boldsymbol{x}) \boldsymbol{\Phi}\right) e\left(\varepsilon_{n, \boldsymbol{k}}(\boldsymbol{x})+i k \Xi_{n, \boldsymbol{k}}(\boldsymbol{x})\right) \\
& +\left(\nabla \tilde{V}_{n, \boldsymbol{k}}(\boldsymbol{x})-i k \tilde{V}_{n, \boldsymbol{k}}(\boldsymbol{x}) \boldsymbol{\Phi}\right) \varepsilon_{S}\left(\nabla V_{n, \boldsymbol{k}}(\boldsymbol{x})+i k V_{n, \boldsymbol{k}}(\boldsymbol{x}) \boldsymbol{\Phi}\right] d \Omega \\
& -\frac{\tilde{V}_{n, \boldsymbol{k}}^{t} V_{n, \boldsymbol{k}}^{t}}{Z(i \omega)}
\end{aligned}
$$


This weak formulation is simply obtained by integrating equation (13), (14) projected onto any test function $\tilde{\boldsymbol{u}}_{n, \boldsymbol{k}}(\boldsymbol{x})$. The boundary integral vanishes as the test functions are chosen so that $\tilde{\boldsymbol{w}}_{n, \boldsymbol{k}}(\boldsymbol{x}-R \boldsymbol{m})=\tilde{\boldsymbol{w}}_{n, \boldsymbol{k}}(\boldsymbol{x})$ on $S_{r}$. For a polyhedron cell, each boundary is generally a polyhedral plane sub-domain that can be associated with a parallel opposite one. The symmetry conditions called $\tilde{\boldsymbol{w}}_{n, \boldsymbol{k}}(\boldsymbol{x}-R \boldsymbol{m})=\tilde{\boldsymbol{w}}_{n, \boldsymbol{k}}(\boldsymbol{x})$ explicitly link these associated surfaces.

\subsection{Numerical computation of the Bloch's waves}

The numerical implementation is obtained by using a standard finite elements method to discretize the weak formulation (16). The assembled matrix equation is given by:

$$
\begin{aligned}
0= & {\left[K\left(Z\left(\omega_{n}\right)\right)+\lambda L\left(\phi, Z\left(\omega_{n}\right)\right)\right.} \\
& \left.-\lambda^{2} H\left(\phi, Z\left(\omega_{n}\right)\right)-\omega_{n}^{2} M\right] \boldsymbol{u}_{n}(k, \phi),
\end{aligned}
$$

where $\lambda=i k, M$ and $K\left(Z\left(\omega_{n}\right)\right)$ are respectively the standard symmetric semi-definite mass and stiffness matrices (the mass matrix is semi definite because elastostatic equation are condensed into the equation), $L\left(\phi, Z\left(\omega_{n}\right)\right)$ is a skew-symmetric matrix and $H\left(\phi, Z\left(\omega_{n}\right)\right)$ is a symmetric semi-definite positive matrix. $\boldsymbol{u}_{n}$ is the generalized eigen vector defined on all degrees of freedom of the used finite element model.

When $k$ and $\phi$ are fixed and $Z$ does not depend on $\omega$ the system (17) is a linear eigen value problem allowing us to compute the dispersion functions $\omega_{n}^{2}(k, \phi)$ and the associated Bloch eigenvector $\boldsymbol{u}_{n}(k, \phi)$.

This approach has been widely used for developing homogenization techniques and spectral asymptotic analysis like in the work of (Allaire \& Congas 1998). It can also be applied for computing wave's dispersion even if Floquet propagators is preferred for 1D or quasi 1D computation, as indicated in (Ichchou, Akrout \& Mencik 2007, Mencik \& Ichchou 2005a). Nevertheless these approaches have been only developed for undamped or lightly damped mechanical systems. In these cases, most of the previously published works present techniques based on the mesh of a real $\boldsymbol{k}$-space (i.e $\boldsymbol{k}$ or $k$ and $\phi$ ) following the boundary of the first Brillouin zone for obtaining the corresponding dispersion curves and the associated Floquet vectors. For undamped system, only propagative or evanescent waves exist corresponding to a family of eigen solutions purely real or imaginary. Discrimination between each class of waves is easy. If a highly damped system $(K, L, H$ are complex frequency dependent) and a frequency dependent electrical shunt impedance are considered, the obtained eigenvalue problem is not quadratic and a complex specific numerical methodology has to be implemented. Furthermore, evanescent parts of propagating waves appear as the imaginary part of $\omega_{n}^{2}(k, \Phi)$. It then becomes much more difficult to distinguish the propagative and evanescent waves as all solution appear complex.

Another much more suitable possibility for computing damped system, dedicated for time/space deconvolution and for computation of diffusion properties as defined by (Collet, Ouisse, Ruzzene \& Ichchou 2011, Collet, Cunefare \& Ichchou 2009, Mencik \& Ichchou 2005a), is to consider the following generalized eigen value problem:

$$
\begin{aligned}
0 & =\left(K \left(Z(\omega)-\omega^{2} M+\lambda_{n}(\omega, \phi) L(\phi, Z(\omega))\right.\right. \\
& \left.-\lambda_{n}^{2}(\omega, \phi) H(\phi, Z(\omega))\right) \boldsymbol{u}_{n}(\omega, \phi)
\end{aligned}
$$

In this problem, the pulsation $\omega$ is a real parameter corresponding to the harmonic frequency. Wave's numbers and Floquet vectors are then computed. An inverse Fourier transformation in the $\boldsymbol{k}$-space domain can lead us to evaluate the physical wave's displacements and energy diffusion operator 
when the periodic distribution is connected to another system as in (Collet et al. 2009). Another temporal inverse Fourier transformation can furnish a way to access spatio-temporal response for non-homogeneous initial conditions. As $L$ is skew-symmetric, the obtained eigen values are quadruple $(\lambda, \bar{\lambda},-\lambda,-\bar{\lambda})$ collapsing into real or imaginary pairs (or a single zero) when all matrices are real (i.e. for an undamped system). In this case a real pair of eigen values correspond to evanescent modes oriented in two opposite directions on the $\boldsymbol{k}$-space and imaginary values to two traveling waves propagating in opposite direction as described in (Garvic 1995).

As previously mentioned, the real part of $\boldsymbol{k}=k \boldsymbol{\Phi}$ vector is restricted to stand inside the first Brillouin zone. In the quadratic eigen value problem (18) nothing restricts computation to only find eigen values satisfying this condition. For direction vector $\boldsymbol{\Phi}$ orthogonal to the lattice facelets (i.e. for $\boldsymbol{\Phi}_{p 1}=[1,0]^{T}$ and $\boldsymbol{\Phi}_{p 2}=[0,1]^{T}$ in bi-dimensional rectangular cell), the periodical conditions expressed for one dimensional wave guide are still valid: if $\lambda_{j}\left(\omega, \boldsymbol{\Phi}_{p}\right)$ is an eigen value associated to $\boldsymbol{w}_{j}\left(\omega, \boldsymbol{\Phi}_{p}\right)$ then $\forall \boldsymbol{m} \in \mathbb{Z}^{3}, \lambda+i . \Phi_{p}^{T}(G . \boldsymbol{m})$ is also an eigen value associated to $\boldsymbol{w}_{j}\left(\omega, \boldsymbol{\Phi}_{p}\right) e^{-i . \boldsymbol{\Phi}_{p}^{T}(G . \boldsymbol{m}) \boldsymbol{x}}$. Thus, for undamped systems, all obtained eigenvalues are periodically distributed in the $\boldsymbol{k}$-space along its principal directions.

\subsection{Computation of the evanescence and damped power flow criteria}

One aim of this paper is to provide a numerical methodology for optimizing the piezoelectric shunt impedance $Z(\omega)$ for controlling energy flow into the periodically distributed piezo-composite structure. For doing this, we need to define suitable criteria evaluating the efficiency of the approach on the features of interest.

The first criterion which is considered for describing the capability of the metacomposite for transmitting structural energy is based on the computation of the wave group velocities. Indeed, they indicate how energy is transported into the considered system and allow to distinguish the 'propagative' and 'evanescent' waves. If a Bloch eigen solution (i.e $\boldsymbol{u}_{n}(\omega, \phi), \boldsymbol{k}_{n}(\omega)$ ) is considered, the associated group velocity vector (Maysenhölder 1994) is given by:

$$
\boldsymbol{C}_{g_{n}}(\omega, \phi)=\nabla_{\boldsymbol{k}} \omega=\frac{\langle\langle\boldsymbol{S}\rangle\rangle}{\left\langle\left\langle e_{t o t}\right\rangle\right\rangle}=\frac{\langle\boldsymbol{I}\rangle}{\left\langle E_{t o t}\right\rangle}
$$

where $\langle\langle:\rangle\rangle$ is the spatial and time average respectively on one cell and one period of time, $\boldsymbol{S}$ is the density of energy flow, $\boldsymbol{I}$ the mean intensity and $e_{t o t}, E_{t o t}$ the total piezomechanical energy and its time average on a period (see (Maysenhölder 1994) for details). In this problem, we only consider mechanical energy transportation as the electrostatic coupling is decentralized on each cell and can not induce spatial energy transportation. The electromechanical coupling effect is also condensed into the mechanical behaviour as explicitly introduced in (Hagood \& von Flotow 1991) and extended to finite element model in (Collet \& Cunefare 2008).

The intensity vector $\boldsymbol{I}$ is expressed as:

$$
\left\langle\boldsymbol{I}_{n}\right\rangle=-\frac{\omega}{2} R e\left(\int_{\Omega_{x}} C\left(\varepsilon_{n}(\boldsymbol{x})+i k \Xi_{n}(\boldsymbol{x})\right) \cdot\left(\boldsymbol{w}_{n}^{*}(\boldsymbol{x})\right) \frac{d \Omega}{V_{o l}}\right)
$$

where.$^{*}$ is the complex conjugate, $R e$ stands for real part and $V_{o l}$ the domain volume.

As the spatio-temporal average of the system Lagragian is null (see (Maysenhölder 1994)), the total energy average is approximated by only computing the kinetic energy average:

$$
\left\langle E_{t o t}\right\rangle=\frac{1}{2 V_{o l}}\left(\int_{\Omega_{x}} \rho \omega^{2} \boldsymbol{w}_{n}(\boldsymbol{x}, \omega, \phi) \cdot \boldsymbol{w}_{n}^{*}(\boldsymbol{x}, \omega, \phi) d \Omega\right) .
$$


The group velocity vectors $\boldsymbol{C}_{g_{n}}(\omega, \phi)$ are computed for all wave numbers at each frequency. In order to focus our analysis on only flexural modes ( $S$ and $S H$ ones) we introduce an indicator allowing to select them by computing the ratio of kinetic energy average on out of plane displacement as:

$$
\operatorname{Ind}(n, \omega, \phi)=\frac{\frac{1}{2 V_{o l}} \int_{\Omega_{x}} \rho \omega^{2} w z_{n}(\boldsymbol{x}) w z_{n}^{*}(\boldsymbol{x}) d \Omega}{\left\langle E_{\text {tot }}\right\rangle},
$$

$w z_{n}(\boldsymbol{x})$ being the $(O z)$ component of vector $\boldsymbol{w}_{n}(\boldsymbol{x})$. Optimization of the shunt impedance $Z(\omega)$ is based on the minimization of the maximal group velocity collinear to the wave number vector (19) for waves having a ratio of transported flexural kinetic energy (22) greater than 0.8. The criterion can then be written as:

$$
\operatorname{Crit}_{1}(Z(\omega), \phi)=\max _{n / \operatorname{Ind}(n, \omega, \phi)>0.8}\left(\boldsymbol{C}_{g_{n}}(\omega, \phi) . \boldsymbol{\Phi}\right) .
$$

The second criterion for optimizing the structural energy absorption is based on the maximization of the damped electric power expressed as the active electrical power

$$
P_{\text {elec }}(n, \omega, \phi)=\frac{1}{2} \operatorname{Re}\left(i \omega Z(\omega) q_{n, \boldsymbol{k}}^{o} q_{n, \boldsymbol{k}}^{o *}\right) .
$$

In order to increase the damping effects inside the smart metacomposite, this term needs to be sufficiently large. In the second case, the criteria is then

$$
\operatorname{Crit}_{2}(Z(\omega), \phi)=\max _{n / \operatorname{Ind}(n, \omega, \phi)>0.8} \frac{1}{P_{\text {elec }}(n, \omega, \phi)} .
$$

\section{Optimization of the flexural energy flow inside the shunted peri-}

\section{odic piezo-composite}

The considered piezo-composite cell is presented in figure 1. The supporting plate material is standard aluminum with $0.1 \%$ of hysteretic damping ratio and the piezoelectric material characteristics are given in table 1.

\subsection{Optimization of the waves group velocities by using Crit $_{1}$}

In this part, optimization of the transmission capability of the designed adaptive metacomposite is considered by using Crit 1 given in equation (23). The objective is to avoid any energy transportation when flexural waves $\left(A_{0}, A_{1}, T H \ldots\right)$ are excited into the periodically distributed shunted piezocomposite cells. The numerical optimization procedure is based on a multidimensional unconstrained nonlinear minimization algorithm (Nelder-Mead). The optimization is done by considering an active/reactive electronic circuit through an impedance $Z(\omega) \in \mathbb{C}$.

The analysis is initialized with an arbitrary complex value of the shunt impedance. Optimization steps are then proceeded using criterion (23) by considering any frequency dependent complex impedance for describing the circuit behaviour. Figure 2 shows the dispersion curves using the propagative parts (here the real one) of the wave numbers $k x_{n}(\omega)$ along $(O x)$ axis, for both initial and optimal impedance shunts. 


\begin{tabular}{||c|c|c||}
\hline \multicolumn{3}{||c||}{ Piezoelectric Material } \\
\hline \hline Symbol & Value & Property \\
\hline$s_{11}^{E}=s_{22}^{E}=s_{33}^{E}$ & $11.6 e^{-12} \mathrm{~Pa}^{-1}$ & \\
$s_{12}^{E}=s_{13}^{E}=s_{23}^{E}$ & $-3.33 e^{-12} \mathrm{~Pa}^{-1}$ & Compliance matrix \\
$s_{44}^{E}=s_{55}^{E}=s_{66}^{E}$ & $45.0 e^{-11} \mathrm{~Pa}^{-1}$ & \\
\hline$\eta$ & $0.1 \%$ & Hysteretic Damping ratio \\
\hline$d_{31}=d_{32}$ & $-6 e^{-11} \mathrm{C} / \mathrm{N}$ & \\
$d_{33}$ & $15.2 e^{-11} \mathrm{C} / \mathrm{N}$ & Piezoelectric matrix \\
$d_{24}=d_{15}$ & $730 e^{-12} \mathrm{C} / \mathrm{N}$ & \\
\hline$\rho$ & $7600 \mathrm{~kg} / \mathrm{m}^{3}$ & Density \\
\hline$\varepsilon_{11}^{T}=\varepsilon_{22}^{T}$ & $504.1 \varepsilon_{o} \mathrm{C} / \mathrm{V} / \mathrm{m}$ & Dielectric Permittivity \\
$\varepsilon_{33}^{T}$ & $270 \varepsilon_{o} \mathrm{C} / \mathrm{V} / \mathrm{m}$ & \\
\hline
\end{tabular}

Table 1: Piezoelectric patch characteristics 


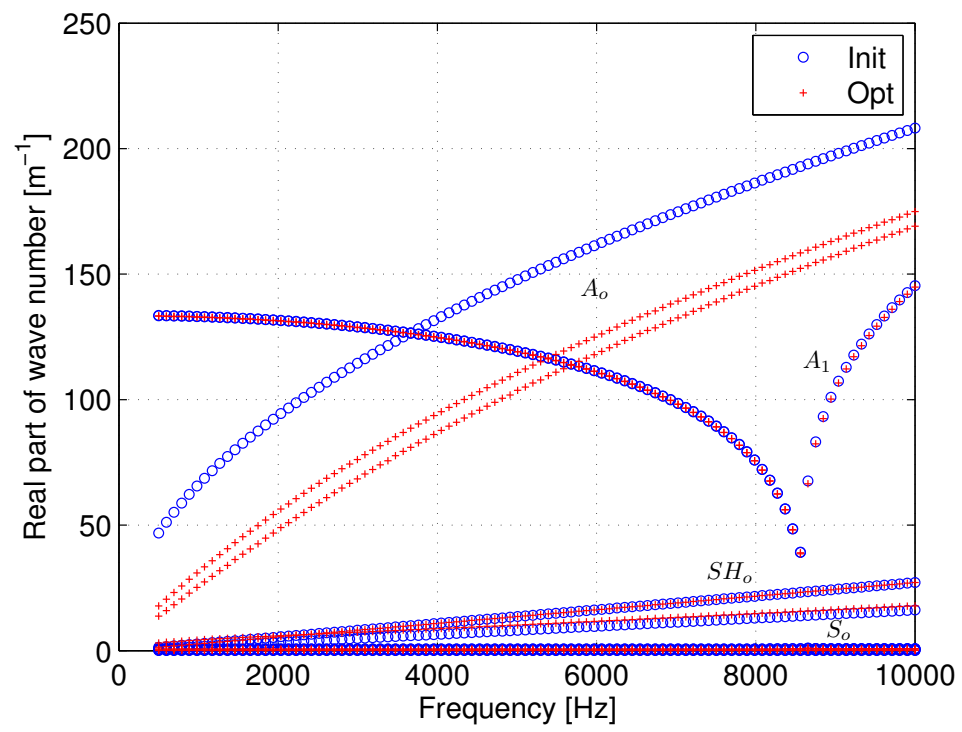

Figure 2: Propagative parts of the wave number $k x_{n}(i \omega)$ along $(O x)$ (all waves included). o: initial

value of shunt; + : optimal value of shunt.

This figure underlines the ability of the procedure to catch all Bloch's solutions including evanescent ones that generally do not appear in literature because of their imaginary character. In the context of structural dynamics, including damping effects is mandatory for real-life applications and all wave numbers become complex, which constitutes a key point in the analysis as underlined in (Collet, Ouisse, Ruzzene \& Ichchou 2011, Garvic 1995).

By using a suitable wave filter based on the group velocity, it is possible to retain for presentation only propagative solutions responsible for energy transport. For a sake of clarity, the following results will be presented by exhibiting only propagative waves. Above results will be filtered and presented as in figure 3, which shows the propagative parts of the wave number $k x_{n}(\omega)$ along $(O x)$. It then becomes easy to observe branches similar to standard $S_{0}, A_{0}, A_{1}, T H$ waves.

The corresponding group velocities along $(O x)$ are presented in figure 4 .

It can immediately be observed that the optimization of the shunt impedance leads to a large decrease of the group velocity of the $A_{0}$ mode while the $A_{1}$ wave, which becomes propagative at 8.8 $k H z$, is not controlled by the optimal configuration (see figure 4). The bending waves also propagate energy with a very slow velocity and can be considered as evanescent. Flexural energy is, also, only transported by the $A_{1}$ mode after the cutting frequency.

The propagative part of the controlled wave is strongly modified, as illustrated in figure 3: from two complex conjugated solutions, the $A_{0}$ wave changes after control to 4 solutions $(2$ complex conjugates and their opposites). This situation is described in (Garvic 1995), it corresponds to high order evanescent waves solutions.

It can also be observed on the group velocity diagram 4 that a non dispersive symmetric wave is also affected by the control whereas it was not targeted by the criterion.

The optimization has been performed for several values of $\phi$. In order to show the stability of the optimized configuration with this angle, the figure 5 shows the propagative parts of the wave number $k x_{n}(\omega)$ along 6 directions forming angles with the $(O x)$ axis of $\frac{n \pi}{20}, n$ being all integers from 0 to 5 . It is clear on this figure that above comments are valid for all angles. In particular, the 


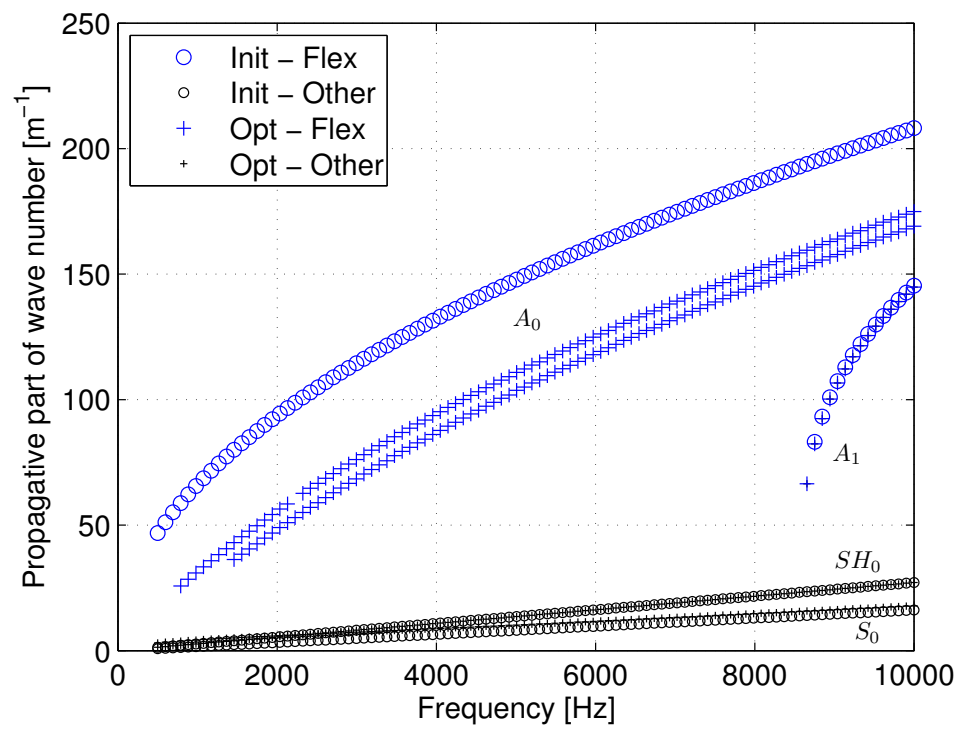

Figure 3: Propagative parts of the wave number $k x_{n}(\omega)$ along $(O x)$ (only propagating waves included). o: initial value of shunt; + : optimal value of shunt. Large marks correspond to flexural waves, small marks correspond to other waves

high order of evanescence of controlled waves in the optimized configurations can be observed in all situations, while some non dispersive symmetric waves are systematically affected by the control whereas they were not targeted by the criterion.

The real and imaginary parts of the optimal impedance are plotted in figure 6 for all angles $\phi$. The optimal impedance values almost correspond to a constant negative capacitance in all directions. The corresponding average value is $-233.66 p C . V^{-1}$. Equivalent resistances corresponding to the active part of the shunt impedance (figure 6) are negative which indicates that the optimization leads to provide energy to the system for controlling mechanical damping effects introduced by hysteretic damping in the model. The optimized configuration tends to converge towards a fully conservative system. The obtained mean value of the resistance is $-1.5319 \Omega$. This is confirmed in figure 7 where the electrical dissipated energy appears negative when the optimal shunt is connected to the patch.

In order to check this point, an optimization run has been performed on a highly damped system, using an hysteretic coefficient of $5 \%$. Figure 8 show the corresponding dispersion curves for $\phi=0$. The optimized configurations leads to a behaviour which is very similar to the one illustrated in figure 3 for lower structural damping value. The same observation can be done for other angle values. This similar behaviour is only related to propagative parts of the wave numbers, while the dissipative effects are modified as illustrated in figure 9 which shows the new optimal impedances. It can be underlined that the electric circuit is fully active with larger resistive parts of average value -74.88 $\Omega$. The observed proportionality between the optimal average resistances $(-1.5319$ and $-74.88 \Omega)$ and the chosen mechanical damping terms $(0.1 \%$ and $5 \%)$ indicates that the optimization leads to annihilate mechanical damping in order to reach a total cancellation of the group velocity of $A_{0}$ mode. The final controlled system is then almost fully reactive.

This last point is of first importance in both physical and technological points of view. Indeed, this results tends to show, on this example, that a pure band gap effect is only reachable if the 


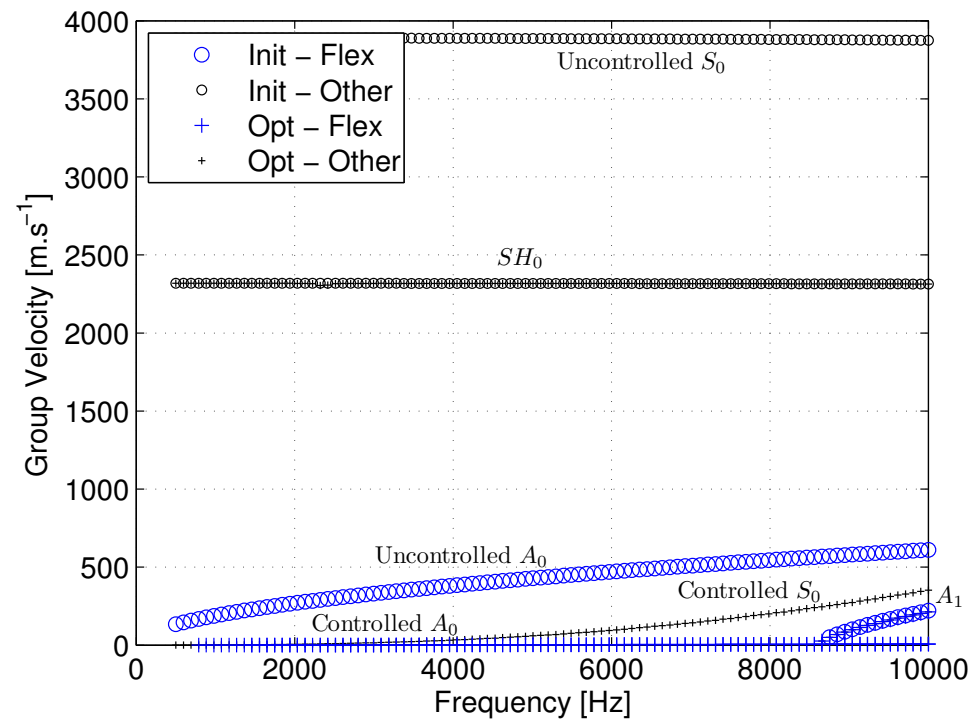

Figure 4: Group velocities along $(O x)$ direction. o: initial value of shunt; +: optimal value of shunt.

Large marks correspond to flexural waves, small marks correspond to other waves

system is completely reactive, which means that no damping occurs in the system. The technological implementation of such interface allowing cancellation of structural transmissibility is then unrealistic since it leads to the implementation of only 'Lyapunov stable' system: the stability margins have to be drastically minimized by using negative resistance for obtaining transmission control.

\subsection{Optimization of damped power flow inside the electric shunts by using $\mathrm{Crit}_{2}$}

Another strategy for optimizing the adaptive metacomposite consists in focusing on the damped power flow inside the electric shunts by using the criteria $\mathrm{Crit}_{2}$ given in equation (25). In this case the objective is to improve the absorption capability of the smart structure.

The propagative parts of the wave number $k x_{n}(i \omega)$ along $(O x)$ axis appear as in figure 10 . The corresponding group velocities along $(O x)$ are presented in figure 11, while the real and imaginary parts of the optimal impedance are plotted in figure 13 and the electrically damped power in figure 12 .

The optimal impedances of the electric shunts are plotted in figure 13 for various values of $\phi$.

The first observation is that the optimization of the shunt impedance for improving the absorption characteristics of the system induces modifications of the group velocities of the controlled waves (see figure 11), while the propagative part of the wave numbers remain unchanged (figure 10). This can be explained by a large improvement of the ratio between the real and imaginary parts of the waves numbers, which physically corresponds to the forcing of propagating effects to increase damping effects: energy can propagate inside the periodically distributed set of active cells for allowing electrical energy conversion. The figure 12 shows that a the dissipated power is largely increased when optimal shunt is connected to the patch.

The optimal impedance values correspond to an almost constant negative capacitance in all directions (see figure 13). The corresponding average value is $-237.43 p C . V^{-1}$. Equivalent resis- 

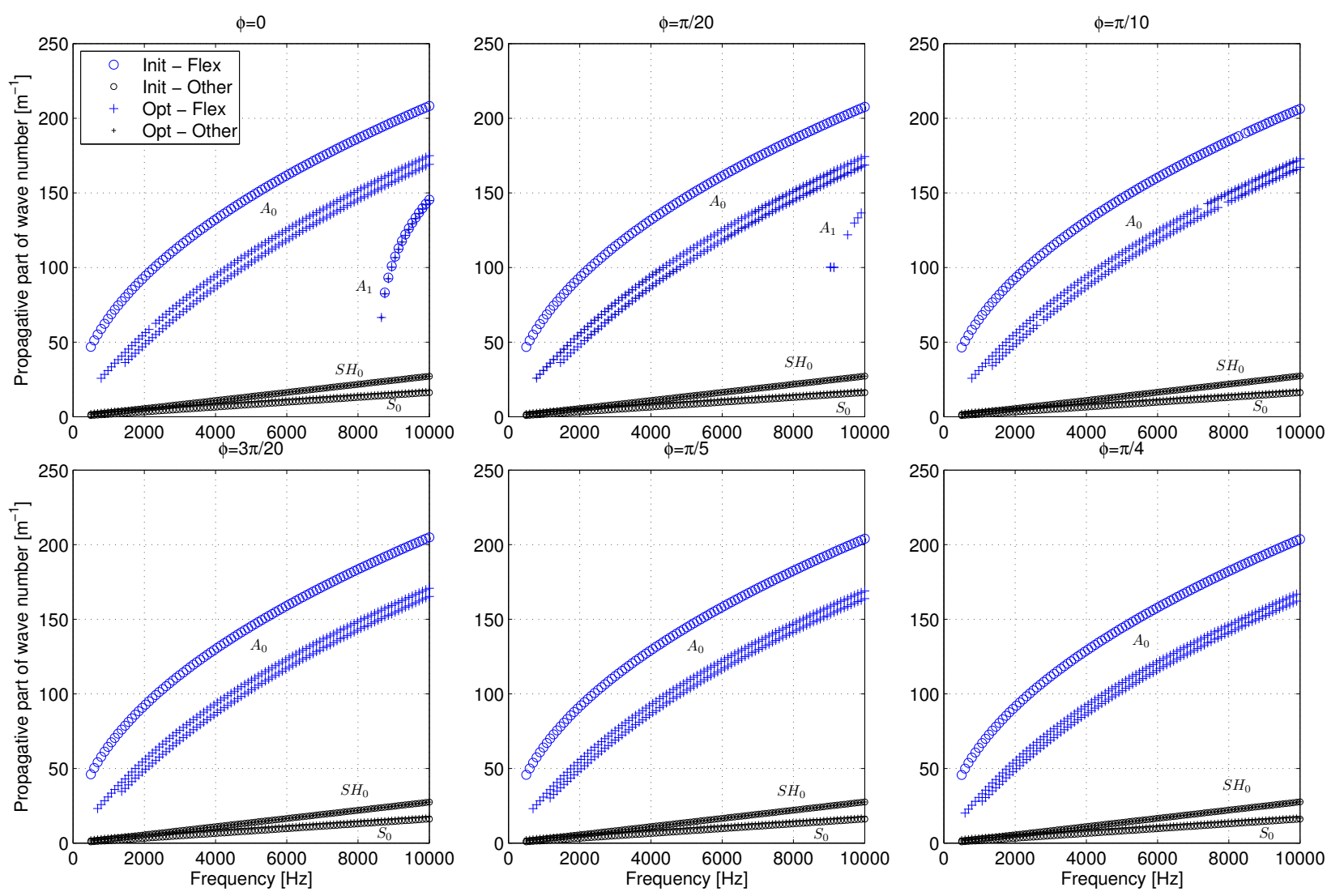

Figure 5: Propagative parts of the wave number $k x_{n}(i \omega)$ along 6 directions forming an angle with

$(O x)$ axis of $\frac{n \pi}{20}$ with $n$ being all integers from 0 to 5 . o: initial value of shunt; + : optimal value of

shunt. Large marks correspond to flexural waves, small marks correspond to other waves

tances corresponding to the active part of the shunt impedance (figure 13) are positive, which is in accordance with the fact that a damping effect is awaited.

\subsection{Validation on a periodically semi-distributed set of adaptive cells}

Up to that point, the optimizations of the smart metacomposite which have been proposed were performed by considering infinite periodic structures. The objective of this section is to illustrate the applicability of the designs for integration into real-life finite structures. The optimal impedance is then applied on a finite set of shunted piezo-composite cells distributed on a part of a totally free plate system submitted to a point force in one corner, as described in figure 14. Material damping is introduced throughout a constant imaginary part of the elasticity tensor of $0.1 \%$. The harmonic response of this system is then computed on the [500,10000] $\mathrm{Hz}$ frequency band when optimal impedances, obtained by criteria $\mathrm{Crit}_{1}$ or $\mathrm{Crit}_{2}$, are connected or not to each patches.

The figure 15 presents the mean kinetic energy on the considered system (i.e on the whole domain $\Omega=\Omega_{s} \cup \Omega_{i} \cup \Omega_{t}$ ) with open circuit and Crit $_{1}$, Crit $_{2}$ optimal shunts connected to each piezoelectric patch. 


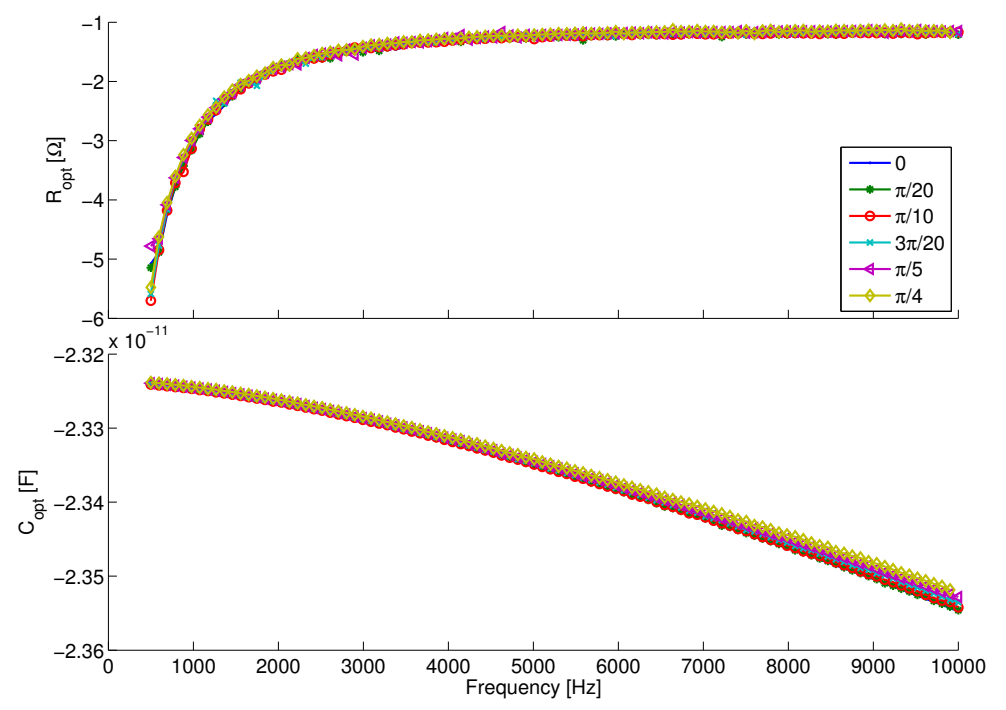

Figure 6: Optimal electric impedance, represented as equivalent resistance and capacitance, obtained

along 6 direction forming an angle with $(O x)$ axis of $\frac{n \pi}{20}$ with $n$ being all integers from 0 to 5 .

From the observation of figure 15, the main particularities of the synthesized optimal impedances can be emphasized. The first configuration, obtained by using $\mathrm{Crit}_{1}$, lead the adaptive interface to avoid any flexural energy transfer. The mechanical response of the system is strongly modified, and the resonance peaks are moved far form the open circuit response, which indicates a modification of the internal dynamics. The second configuration, based on $\mathrm{Crit}_{2}$ optimal shunt, forces energy dissipation into the resistive part of the electric shunt. In that case, the conservation of the modal architecture into the energy spectrum can be observed, even if some damping effects limit the peak's amplitudes.

To go further, one can take a closer look at the energy repartition into the considered system in figure 16, which shows the ratio of the kinetic energy finally transmitted to the sub-domain $\Omega_{t}$. The minimization of the flexural wave group velocity $\left(\right.$ Crit $\left._{1}\right)$ leads to decrease the part of kinetic energy transmitted to the non-excited part of the plate located behind the adaptive interface while the maximization of the damped energy $\left(\mathrm{Crit}_{2}\right)$ only slightly modify this distribution. The modification of the energy distribution into the system is very important after $6 \mathrm{kHz}$ and increase with the frequency when the energy is globally transported by $A_{0}$ mode. This is not the case in the lower frequency band when the interface works in the near field domain of the applied point force.

The figure 17 shows the corresponding results in terms of velocity root mean square.

These numerical results clearly show a strong influence of the modifications of the wave dispersion characteristics on the standing wave responses plotted in figure 17 especially when $\mathrm{Crit}_{1}$ is used. In particular, at $2550 \mathrm{~Hz}$, a large attenuation of the dynamic response of the system can be observed when optimal shunts are connected. In this case, when the shunts are optimized for cancelling flexural energy flow $\left(\mathrm{Crit}_{1}\right)$, the location of the nodal line are also modified. With an optimization aiming at damping energy in the electric resistance ( $\mathrm{Crit}_{2}$ ), only to a standard attenuation can be observed. At $8000 \mathrm{~Hz}$ when $\mathrm{Crit}_{1}$ optimal shunts are connected, a large part of the system energy remains in its left part where the applied force is located. The smart metacomposite interface also changes the system admittance and filter wave diffusion by increasing is reflexivity properties. The energy diffusion is also clearly condensed into the left part of the system with a largely decreased 


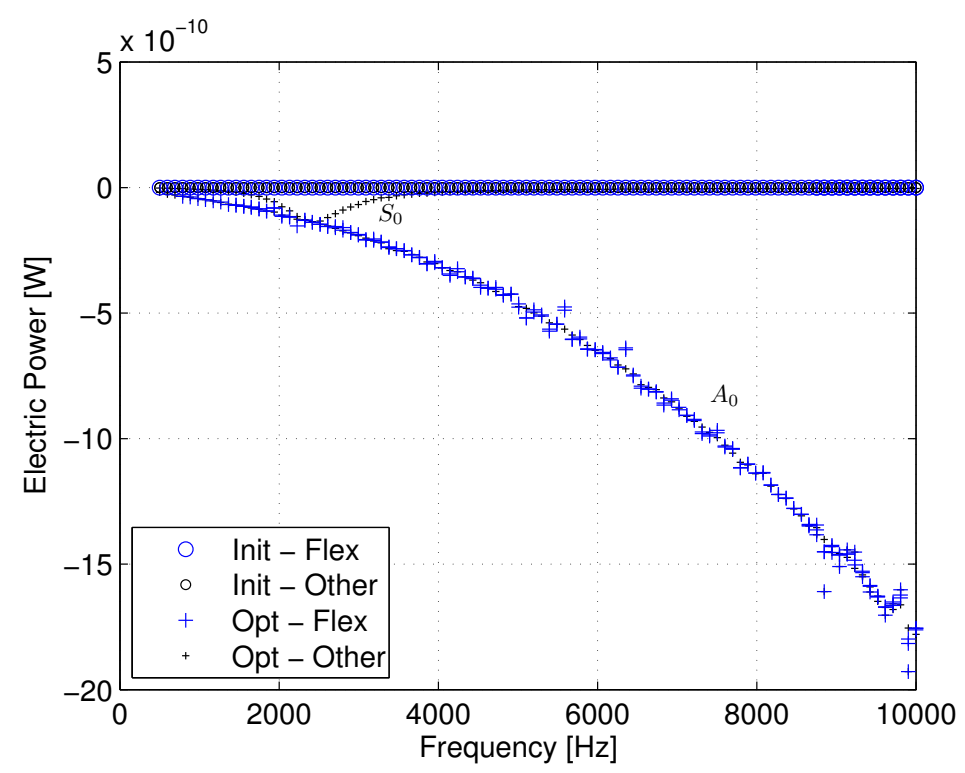

Figure 7: Damped power inside the electric circuit. o: initial value of shunt; + : optimal value of

shunt. Large marks correspond to flexural waves, small marks correspond to other waves

amplitude compared to the one obtained with open circuit and Crit $_{2}$ optimal shunt. With this type of shunt something similar to a wave trap effect can be observed. With $\mathrm{Crit}_{2}$ optimal shunt, an improvement of the damping effect leading to vibration attenuation can be observed, without wave trap behaviour.

\section{Conclusions}

This article presents a numerical procedure able to compute the damped wave's dispersion functions in the whole first Brillouin domain of multi dimensional piezo-elastodynamical wave guides. The method was applied for determining the optimal shunt impedance allowing the minimization of the group velocities of the flexural waves and the damping capability of the distributed system. The smart metacomposite can then be included in a finite structure to confer on it new functionalities. Some numerical tests on a finite dimension system incorporating a semi-distributed set of shunted piezo-composite cells has been performed. A strong influence of the designed shunt circuits has been underlined in the dynamical response of the system and energy distribution into each structural sub domains. Even if the link between the obtained wave properties are not clearly established especially in the low frequency range where the adaptive interface is located in the near field domain of the input force, it has been demonstrated that the numerical procedures can be used for optimizing the energy diffusion operator of such adaptive mechanical interface. To do so, additional work has to be done for optimizing the complete interface scattering and for controlling the evanescent waves playing an important role in the finite system dynamical response in the low frequency band.

The proposed methodology can also be used for studying particular dissipation phenomenon such as those induced by complex shunted piezoelectric patches as proposed by (Beck, Cunefare \& Ruzzene 2008b) and (Casadei et al. 2009), or even foams or complex polymers behaviours. The 


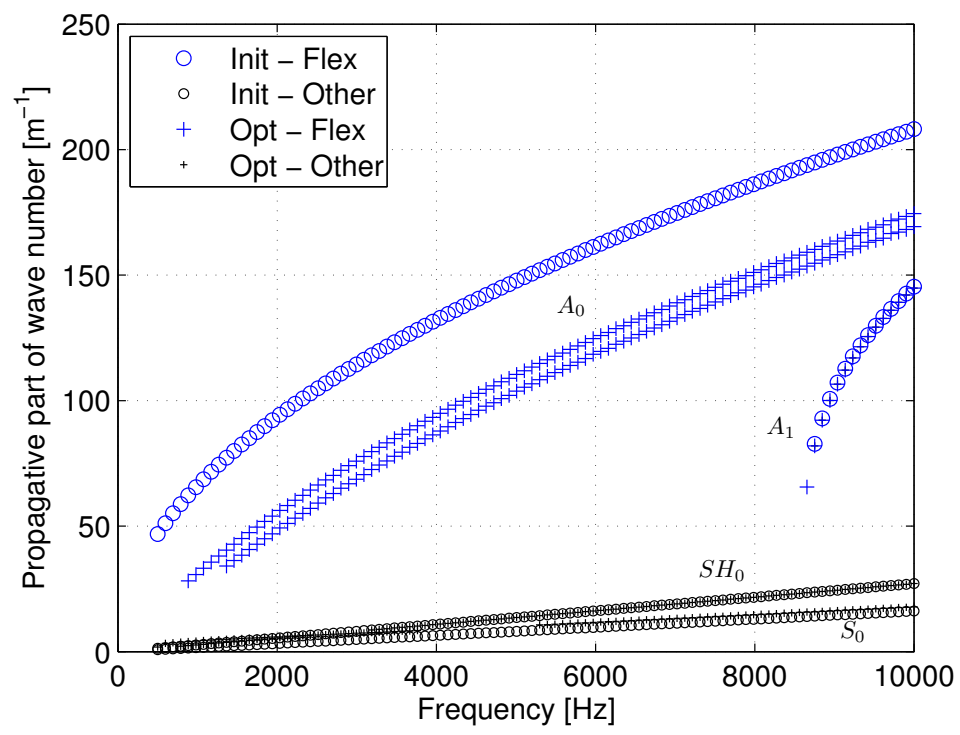

Figure 8: Propagative parts of the wave number $k x_{n}(i \omega)$ along $(O x)$, for high hysteretic damping of $5 \%$ (only propagating waves included). o: initial value of shunt; + : optimal value of shunt. Large marks correspond to flexural waves, small marks correspond to other waves

proposed method furnishes an efficient tool for future optimization of distributed smart cells as proposed in the case of 1D wave guide by (Collet et al. 2009).

\section{References}

\section{References}

Aalami, B. (1973). Waves in prismatic guides of arbitrary cross section, Journal of Applied Mechanics 40(4): 1067-1072.

Agnes, G. (1994). Active/passive piezoelectric vibration suppression, in C. D. Johnson (ed.), Proceedings of SPIE Smart Structures and Materials, pp. 24-34.

Allaire, G. \& Congas, C. (1998). Bloch waves homogenization and spectral asymptotic analysis, Journal de Mathématiques Pures et Appliquées 77: 153-208.

Banks, H., Smith, R. \& Wang, Y. (1996). Smart material structures: Modeling Estimation and Control, Masson and Wiley.

Bartoli, I., Marzani, A.and Lanza Di Scalea, F. \& Viola, E. (2006). Modeling wave propagation in damped waveguides of arbitrary cross-section, Journal of Sound and Vibration 295: 685-707. 


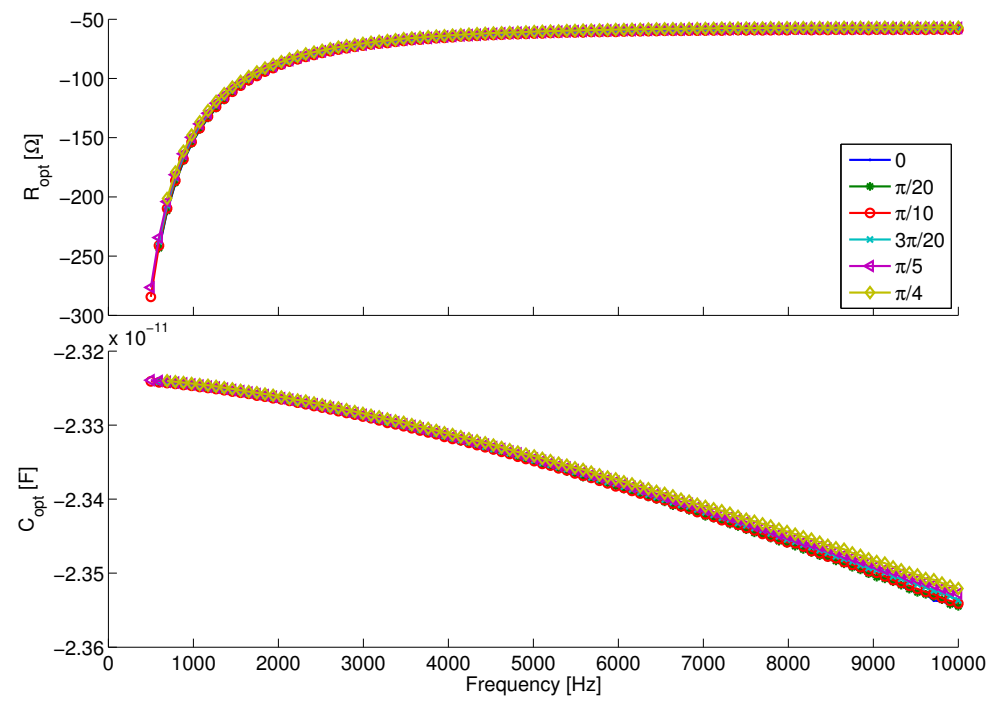

Figure 9: Optimal electric impedance for high hysteretic damping of $5 \%$, represented as equivalent resistances and capacitances, obtained along 6 direction forming an angle with $(O x)$ axis of $\frac{n \pi}{20}$ with $n$ being all integers from 0 to 5 .

Baz, A. (1997). Optimization of energy dissipation characteristics of active constrained layer damping, Smart Materials and Structures 6(3): 360.

Beck, B., Cunefare, K. \& Ruzzene, M. (2008a). Broadband vibration suppression assessment of negative impedance shunts, Proceedings of ASME - SMASIS.

Beck, B., Cunefare, K. \& Ruzzene, M. (2008b). Broadband vibration suppression assessment of negative impedance shunts, Proceedings of ASME - SMASIS.

Becker, J., Fein, O., Maess, M. \& Gaul, L. (2006). Finite element-based analysis of shunted piezoelectric structures for vibration damping, Computers and Structures 84(31-32): 2340-2350.

Behrens, S., Fleming, A. \& Moheimani, S. (2001). New method for multiple-mode shunt damping of structural vibration using a single piezoelectric transducer, in D. Inman (ed.), Proceedings of SPIE Smart Structures and Materials, pp. 239-250.

Bensoussan, A., Lions, J. \& Pananicolaou, G. (1978). Asymptotic Analysis for Periodic Structures, North Holland.

Bloch, F. (1928). Über die Quantenmechanik der Electron in Kristallgittern, Zeitschrift für Physik 52: $550-600$.

Bocquillet, A., Ichchou, M. N. \& Jezequel, L. (2003). Energetics of axisymmetric fluid-filled pipes up to high frequencies, Journal of Fluids and Structures 17: 491-510.

Casadei, F., Beck, B., Ruzzene, M. \& Cunefare, K. (2009). Vibration control of plates featuring periodic arrays of hybrid shunted piezoelectric patches, Proceedings of SPIE Smart Structures and Materials. 


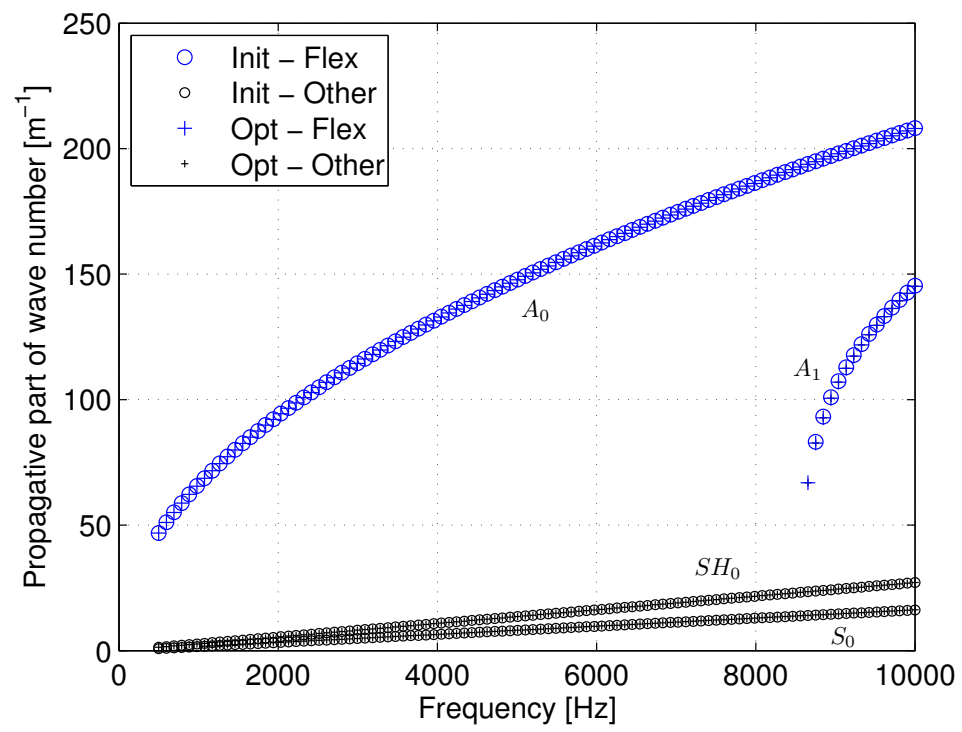

Figure 10: Propagative parts of the wave number $k x_{n}(i \omega)$ along $(O x)$, for high hysteretic damping of $5 \%$ (only propagating waves included). o: initial value of shunt; + : optimal value of shunt. Large marks correspond to flexural waves, small marks correspond to other waves

Chen, F. \& Wilcox, P. (2007). The effect of load on guided wave propagation, Ultrasonics 47(14): $111-122$.

Collet, M. \& Cunefare, K. (2008). Modal Synthesis and Dynamical Condensation Methods for Accurate Piezoelectric Systems Impedance Computation, Journal of Intelligent Material Systems and Structures 19(11): 1251-1271.

Collet, M., Cunefare, K. \& Ichchou, M. (2009). Wave Motion Optimization in Periodically Distributed Shunted Piezocomposite Beam Structures, Journal of Intelligent Material Systems and Structures 20(7): 787-808.

Collet, M., Ouisse, M., Ichchou, M. \& Ruzzene, M. (2010). Numerical tools for semi-active optimization of $2 \mathrm{~d}$ wave's dispersion into mechanical system, Proceedings of ASME-SMASIS.

Collet, M., Ouisse, M., Ichchou, M. \& Ruzzene, M. (2011). Semi-active optimization of 2d wave's dispersion into mechanical systems by the mean of periodically distributed shunted piezoelectric patches: a new class of adaptive metamaterials,, Proceedings of SPIE Smart Structures/NDE.

Collet, M., Ouisse, M., Ruzzene, M. \& Ichchou, M. (2011). Floquet-bloch decomposition for the computation of dispersion of two-dimensional periodic, damped mechanical systems, International Journal of Solids and Structures 48(20): 2837-2848.

Corr, L. \& Clark, W. (2002). Comparison of lowfrequency piezoelectric switching shunt techniques for structural damping, Journal of Smart Material and Structrues 11(3): 370-376.

Cross, C. \& Fleeter, S. (2002). Shunted piezoelectrics for passive control of turbomachine blading flow-induced vibrations, Smart Material Structures 11: 239-248. 


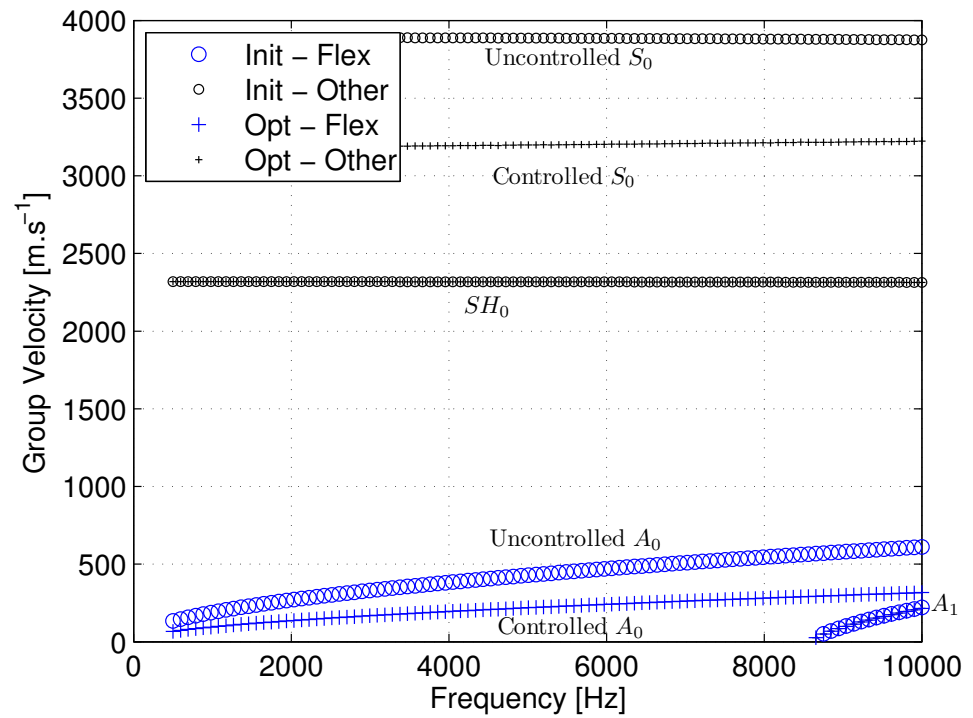

Figure 11: Group velocities along $(O x)$ direction. o: initial value of shunt; +: optimal value of shunt. Large marks correspond to flexural waves, small marks correspond to other waves

Cunefare, K. (2006). Negative capacitance shunts for vibration suppression: wave based tuning and reactive input power, Proceedings of Active 2006.

Damljanovic, V. \& Weaver, R. L. (2004). Propagating and evanescent elastic waves in cylindrical waveguides of arbitrary cross section, Journal of the Acoustical Society of America 115: 15721581.

dell'Isola, F., Maurini, C. \& Porfiri, M. (2004). Passive damping of beam vibrations through distributed electric networks and piezoelectric transducers: prototype design and experimental validation, Smart Material Structures 13: 299-308.

Den Hartog, J. (1956). Mechanical vibrations, 4th edn, McGraw-Hill, New-York.

Duhamel, D., Mace, B. \& Brennan, M. (2006). Finite element analysis of the vibrations of waveguides and periodic structures, Journal of Sound and Vibration 294(1-2): 205-220.

Duhamel, E. (2007). Finite element computation of green's functions, Engineering Analysis with Boundary Elements 31: 919-930.

Finnveden, S. (2004). Evaluation of modal density and group velocity by a finite element method, Journal of Sound and Vibration 273: 51-75.

Finnveden, S. \& Fraggstedt, M. (2008). Waveguide finite elements for curved structures, Journal of Sound and Vibration 312(4-5): $644-671$.

Fleming, A., Behrens, S. \& Moheimani, S. (2003). Reducing the inductance requirements of piezoelectric shunt damping systems, Smart Materials and Structures 12(1): 57-64.

Fleming, A., Belirens, S. \& Moheimani, S. (2000). Synthetic impedance for implementation of piezoelectric shunt-damping circuits, Electronics Letters 36(18): 1525-1526. 


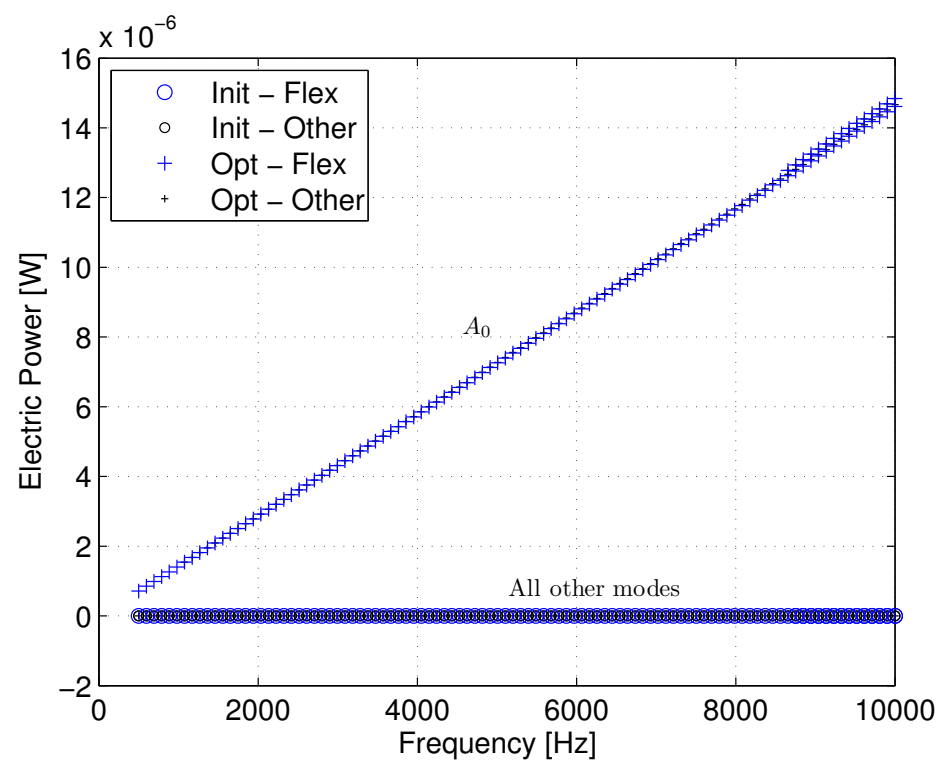

Figure 12: Damped power inside the electric circuit. o: initial value of shunt; + : optimal value of shunt. Large marks correspond to flexural waves, small marks correspond to other waves

Fleming, A. J. \& Moheimani, S. O. R. (2004). Improved Current and Charge Amplifiers for Driving Piezoelectric Loads, and Issues in Signal Processing Design for Synthesis of Shunt Damping Circuits, Journal of Intelligent Material Systems and Structures 15(2): 77-92.

Floquet, G. (1883). Sur les équations différentielles linéaires à coefficients périodiques, Annales de l'Ecole Normale Supérieure 12: 47-88.

Forward, R. (1978). Electromechanical transducer-coupled mechanical structure with negative capacitance compensation circuit, Patent US4158787.

Fukada, E., Date, M. \& Kimura, K. (2002). Sound isolation by piezoelectric polymer films connected with negative capacitance circuits, Proceedings of 11th International Symposium on Electrets .

Garvic, L. (1995). Computation of propagative waves in free rail using a finite element technique, Journal of Sound and Vibration 185: 531-543.

Gavric, L. (1995). Computation of propagatives waves in free rail using a finite element technique, Journal of Sound and Vibration 185(3): 531-543.

Hagood, N. \& von Flotow, A. (1991). Damping of structural vibrations with piezoelectric materials and passive electrical networks, Journal of Sound and Vibration 146(2): 243-268.

Hardy, P., Ichchou, M., Jezequel, L. \& Trentin, D. (2009). A hybrid local energy formulation for plates mid-frequency flexural vibrations, European Journal of Mechanics - A/Solids 28(1): 121 -130 .

Hayashi, T., Song, W. \& Rose, J. (2003). Guided wave dispersion curves for a bar with an arbitrary cross-section, a rod and rail example, Ultrasonics 41(3): 175-183. 


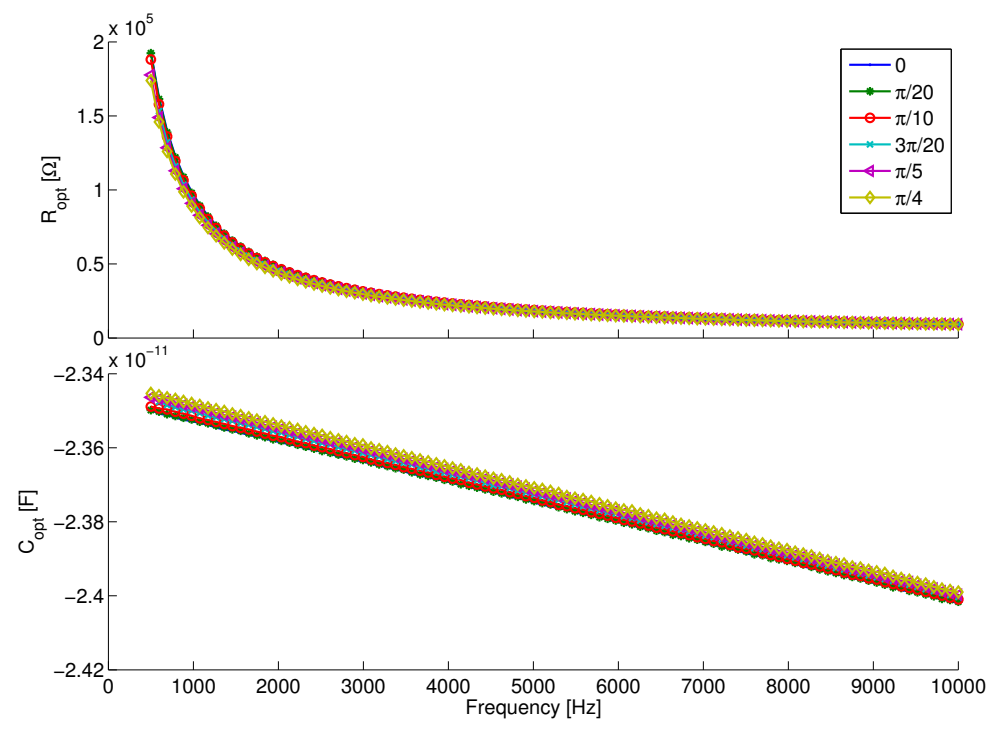

Figure 13: Optimal electric impedance, represented as equivalent resistances and capacitances, obtained along 6 direction forming an angle with $(O x)$ axis of $\frac{n \pi}{20}$ with $n$ being all integers from 0 to 5.

Houillon, L., Ichchou, M. \& Jezequel, L. (2005). Wave motion in thin-walled structures, Journal of Sound and Vibration 281(3-5): 483-507.

Hurlebaus, S. \& Gaul, L. (2006). Smart structure dynamics, Mechanical System and Signal Processing 20: 255-281.

Ichchou, M., Akrout, S. \& Mencik, J. (2007). Guided waves group and energy velocities via finite elements, Journal of Sound and Vibration 305(4-5): 931-944.

Joannopoulos, J., Meade, R. \& Winn, J. (1995). Photonic Crystals: Molding the Flow of Light, Princeton University Press.

Kittel, C. (1986). Introduction to Solid State Physics, John Wiley and Sons, New York.

Mace, B., Duhamel, D., Brennan, M. \& Hinke, L. (2005). Finite element prediction of wave motion in structural waveguides, Journal of Acoustical Society of America 117(5): 2835-2843.

Maess, M., Wagner, N. \& Gaul, L. (2006). Dispersion curves of fluid filled elastic pipes by standard fe models and eigenpath analysis, Journal of Sound and Vibration 296(1-2): 264 - 276.

Maysenhölder, W. (1994). Körperschall-energie Grundlagen zur Berechnung von Energiedichten und Intensitäten, Wissenschaftliche Verlagsgesellschaft, Stuttgart.

Mead, D. (1973). A general theory of harmonic wave propagation in linear periodic systems with multiple coupling, Journal of Sound and Vibration 27: 235-260.

Mencik, J. \& Ichchou, J. (2005a). Multi-mode propagation and diffusion in structures through finite elements, European Journal of Mechanics A-Solids 24(5): 877-898. 


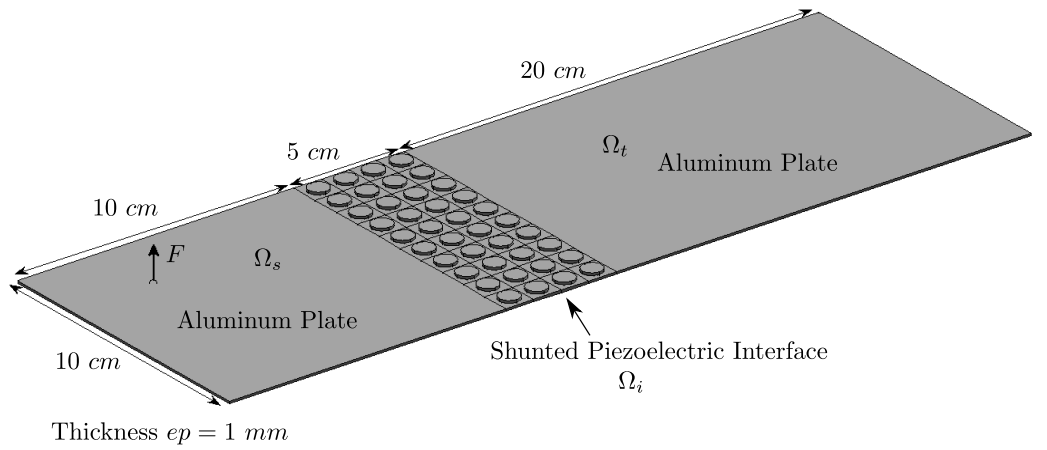

Figure 14: 3D piezocomposite periodic semi-distributed cells as plate interface

Mencik, J. \& Ichchou, M. (2005b). Multi-mode propagation and diffusion in structures through finite elements, European Journal of Mechanics - A/Solids 24(5): 877-898.

Mencik, J. \& Ichchou, M. (2007). Wave finite elements in guided elastodynamics with internal fluid, International Journal of Solids and Structures 44: 2148-2167.

Milman, M. H. \& Cheng-Chih, C. (1994). Optimization methods for passive damper placement and tuning, J. of guid. cont. and dyn. 17(4): 848-856.

Moheimani, S. \& Fleming, A. (2006). Piezoelectric transducers for vibration control \& damping, (Advances in industrial control), Springer.

Nelson, P. \& Elliott, S. (1992). Active Control of Sound, Pub. Academic Press, London, San Diego.

Nelson, R. \& Dong, S. (1973). High frequency vibrations and waves in laminated orthotropic plates, Journal of Sound and Vibration 30(1): 33 - 44.

Neubauer, M., Oleskiewicz, R., Popp, K. \& Krzyzynski, T. (2006). Optimization of damping and absorbing performance of shunted piezo elements utilizing negative capacitance, Journal of Sound and Vibration 298(1-2): $84-107$.

Ormondroyd, J. \& Den Hartog, J. (1928). The theory of dynamic vibration absorber, Trans., ASME APM-50-7: 9-22.

Park, C. \& Baz, A. (2005). Vibration control of beams with negative capacitive shunting of interdigital electrode piezoceramics, Journal of Vibration and Control 11: 331-346.

Park, J. \& Palumbo, D. (2004). A new approach to identify optimal of shunting elements for maximum damping of structural vibration using piezelectrical patches, Active 2004, Institute of Noise Control Engineers.

Preumont, A. (2002). Vibration control of active structures: an introduction, Springer.

Spencer, B. F. \& Nagarajaiah, S. (2003). State of the art of structural control, J. Struct. Eng. 129(7): 1-10. 


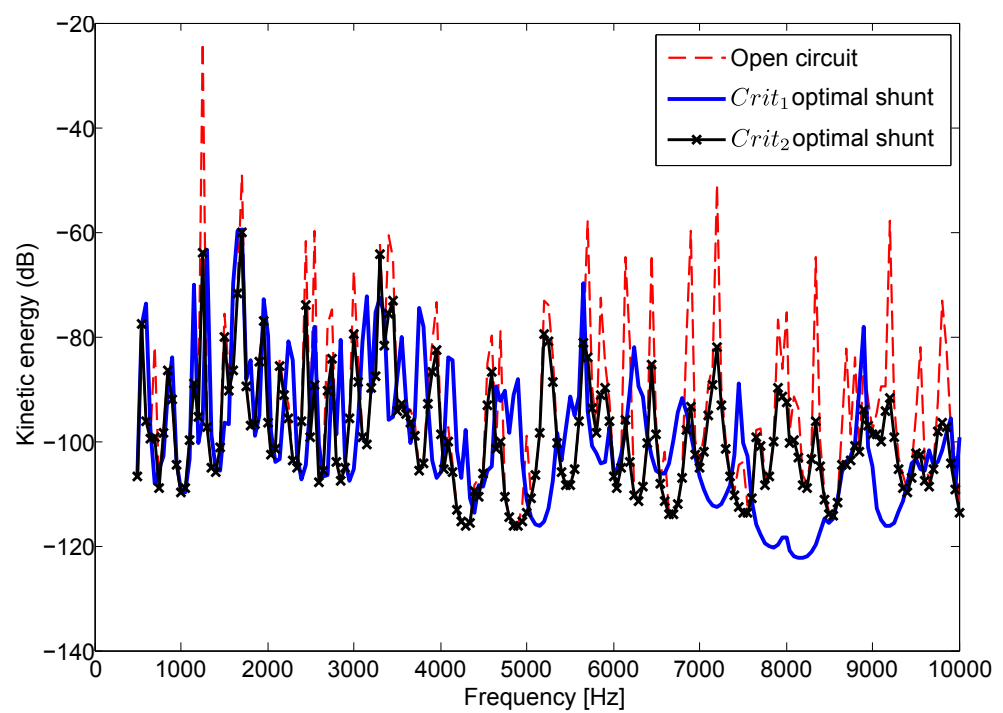

Figure 15: Mean value of the kinetic energy on the whole domain $\Omega$ with open circuit (red dashed line), Crit $_{1}$ (blue line) and Crit $_{2}$ (black marked line) optimal shunt connected to piezoelectric patches

Thorp, O., Ruzzene, M. \& Baz, A. (2001). Attenuation and localization of wave propagation in rods with periodic shunted piezoelectric patches, Smart Materials and Structures 10: 979-989.

Thorp, O., Ruzzene, M. \& Baz, A. (2005). Attenuation of wave propagation in fluid-loaded shells with periodic shunted piezoelectric rings, Smart Materials and Structures 14: 594-604.

Wu, S. (1998). Method for multiple-mode shunt damping of structural vibration using a single pzt transducer, Proceedings of SPIE Smart Structures and Materials.

Wu, S. \& Bicos, A. (1997). Structural vibration damping experiments using improved piezoelectric shunts, in L. P. Davis (ed.), Proceedings of SPIE Smart Structures and Materials, pp. 40-50.

Zhou, W., Ichchou, M. \& Mencik, J. (2009). Analysis of wave propagation in cylindrical pipes with local inhomogeneities, Journal of Sound and Vibration 319(1-2): 335-354. 


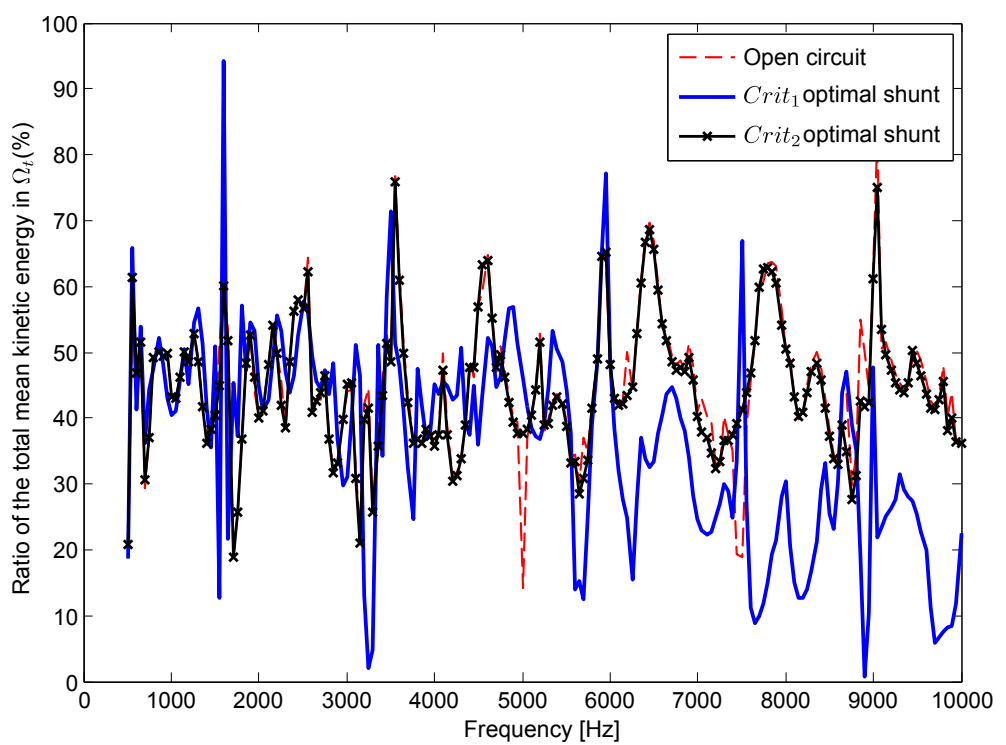

Figure 16: $\Omega_{t}$ ratio of the total kinetic energy mean value in percent with open circuit (red dashed line), Crit $_{1}$ (blue line) and Crit $_{2}$ (black marked line) optimal shunt connected to piezoelectric patches 

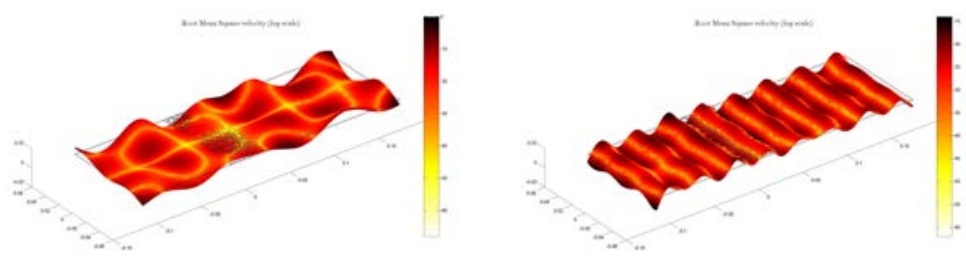

(a) Open circuit patches

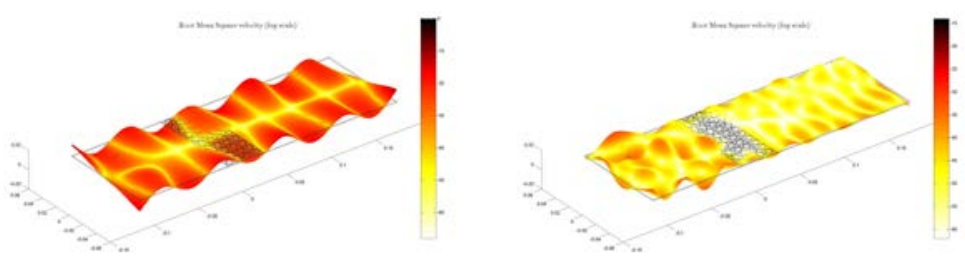

(b) Crit $_{1}$ Optimal Shunt

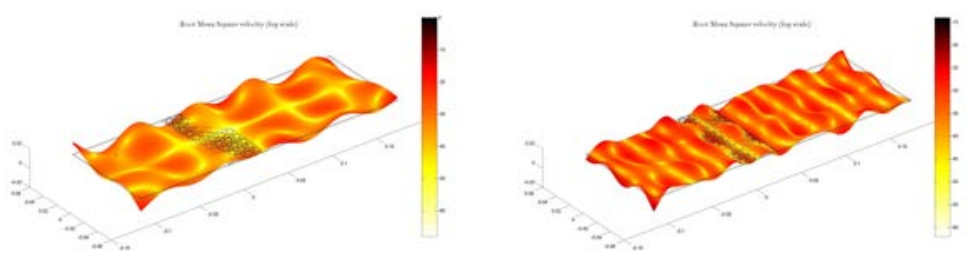

(c) $\mathrm{Crit}_{2}$ Optimal Shunt

Figure 17: Velocity root mean square level of the system submitted to an external point force at $2550 \mathrm{~Hz}$ (left) and $8000 \mathrm{~Hz}$ (right) for all piezoelectric cells in open circuit (a), connected to optimal shunt impedance obtained with $\mathrm{Crit}_{1}$ (b) and $\mathrm{Crit}_{2}$ (c) 